\title{
A Li-Yau inequality for the 1-dimensional Willmore energy
}

\author{
Marius Müller* and Fabian Rupp ${ }^{\dagger}$
}

March 2, 2021

\begin{abstract}
By the classical Li-Yau inequality, an immersion of a closed surface in $\mathbb{R}^{n}$ with Willmore energy below $8 \pi$ has to be embedded. We discuss analogous results for curves in $\mathbb{R}^{2}$, involving Euler's elastic energy and other possible curvature functionals. Additionally, we provide applications to associated gradient flows.
\end{abstract}

Keywords: Li-Yau inequality, Willmore functional, elastic energy, embeddedness. MSC(2020): $53 \mathrm{~A} 04$ (primary), 49Q10, 53E40 (secondary).

\section{Introduction and main results}

For an immersion $f: \Sigma \rightarrow \mathbb{R}^{n}$ of a surface $\Sigma$, its Willmore energy is defined by

$$
\mathcal{W}(f):=\frac{1}{4} \int_{\Sigma}|H|^{2} \mathrm{~d} \mu .
$$

Here $H$ denotes the mean curvature vector and $\mu$ is the Riemannian measure induced by pulling back the Euclidean metric to $\Sigma$. In their fundamental work [LY82, Li and Yau proved an inequality which yields that an immersion with not too large Willmore energy must in fact be an embedding. More specifically, if $\Sigma$ is compact, then

$$
\mathcal{W}(f)<8 \pi \text { implies that } f \text { is an embedding. }
$$

Moreover, as a doubly covered round sphere shows, the constant $8 \pi$ in (2) is optimal.

\footnotetext{
*Mathematisches Institut, Albert-Ludwigs-Universität Freiburg, 79104 Freiburg im Breisgau, Germany. marius.mueller@math.uni-freiburg.de

${ }^{\dagger}$ Institute of Applied Analysis, Ulm University, Helmholtzstraße 18, 89081 Ulm, Germany. fabian.rupp@uni-ulm.de
} 
In this article, we study the question whether an analogous result as in (2) is true for planar curves. For a closed smooth curve $\gamma: \mathbb{S}^{1} \rightarrow \mathbb{R}^{2}$, which is immersed, i.e. $\left|\gamma^{\prime}\right|>0$, and has signed curvature $\kappa$, its elastic energy is defined by

$$
\mathcal{E}(\gamma):=\int_{\mathbb{S}^{1}}|\kappa|^{2} \mathrm{~d} s
$$

This formally resembles the Willmore energy. However, in contrast to (1), $\mathcal{E}$ is not scaling invariant, whereas the property of being embedded is. A natural scaling invariant onedimensional version of the Willmore energy is the total curvature, defined by

$$
\mathcal{K}(\gamma):=\int_{\mathbb{S}^{1}}|\kappa| \mathrm{d} s
$$

It has a wide range of geometric applications and has been studied extensively, for instance in Fen29, Fár49, Mil50, Mil53.

We will show that the total curvature does not allow for a non-trivial version of (2). This will be a consequence of the following observation.

Theorem 1.1. We have

$$
\begin{aligned}
2 \pi & =\inf \left\{\mathcal{K}(\gamma) \mid \gamma \in C^{2}\left(\mathbb{S}^{1} ; \mathbb{R}^{2}\right) \text { non-embedded immersion }\right\} \\
& =\inf \left\{\mathcal{K}(\gamma) \mid \gamma \in C^{2}\left(\mathbb{S}^{1} ; \mathbb{R}^{2}\right) \text { immersion }\right\} .
\end{aligned}
$$

Moreover, the infimum among non-embedded immersions is not attained.

In order to obtain a non-trivial version of (2) we need to identify a different quantity. Our main result shows that the elastic energy provides a positive answer, when restricted to curves of fixed length, or - equivalently - multiplied with the length functional $\mathcal{L}$.

Theorem 1.2 (Main theorem). If $\gamma \in W^{2,2}\left(\mathbb{S}^{1}, \mathbb{R}^{2}\right)$ is an immersed curve with

$$
\mathcal{E}(\gamma) \mathcal{L}(\gamma)<c^{*}:=\mathcal{E}\left(\gamma^{*}\right) \mathcal{L}\left(\gamma^{*}\right),
$$

then $\gamma$ is an embedding. Here $\gamma^{*}$ is the figure eight elastica (see Definition 5.1).

Remark 1.3. The value of $c^{*}$ is sharp since $\gamma^{*}$ itself is not an embedding, cf. Lemma 5.6 for the details. A numerical computation yields $c^{*} \simeq 112.4396$.

We have thus identified a geometric quantity of curves whose smallness ensures that the curve is embedded.

Note that it was already observed in Woj20, Lemma 2.1], that if $\gamma \in W^{2,2}\left(\mathbb{S}^{1} ; \mathbb{R}^{n}\right)$ has a point with multiplicity $k \in \mathbb{N}$, then $\mathcal{E}(\gamma) \mathcal{L}(\gamma)>\pi^{2} k^{2}$. Such a relation between multiplicity and energy is also part of the statement of the original Li-Yau inequality for the Willmore energy [LY82]. However, we remark that the bound in Woj20 cannot be used to ensure embeddedness, since any curve $\gamma$ with $\mathcal{E}(\gamma) \mathcal{L}(\gamma) \leq 4 \pi^{2}$ has to be a one-fold covered circle, cf. Lemma 5.4. Moreover, with the methods of geometric 
measure theory, in [Poz20, (16)] the bound $k \leq \frac{1}{2} \mathcal{K}(\gamma)$ was established. As $\mathcal{K}(\gamma) \geq 2 \pi$, this estimate cannot answer the question of embeddedness either.

The idea for proving Theorem 1.2 is to look at the variational problem

$$
\inf \left\{\mathcal{E}(\gamma) \mathcal{L}(\gamma) \mid \gamma \in W^{2,2}\left(\mathbb{S}^{1} ; \mathbb{R}^{2}\right) \text { non-embedded immersion }\right\}
$$

Minimizing among non-embedded immersions is a non-standard condition, because the admissible set is not open. This causes difficulties in applying Euler-Lagrange methods. However, we will be able to deduce that the minimizer is an interior point of the admissible set and thus satisfies an Euler-Lagrange equation. This can be achieved by a detailed analysis of the self-intersections of minimizers. The main ingredient here is the classification of planar elastic curves (see for instance [LS84c, DHMV08, DP17]).

As a future extension of Theorem 1.2 one could also try to find such embeddednessensuring quantities in other ambient manifolds than $\mathbb{R}^{2}$.

In the hyperbolic halfplane $\mathbb{H}^{2}$ an embeddedness-ensuring quantity can indeed be identified. By [LS84a] one has for all immersed curves $\gamma \in C^{\infty}\left(\mathbb{S}^{1} ; \mathbb{H}^{2}\right)$

$$
\int_{\mathbb{S}^{1}}\left|\kappa_{\mathbb{H}}[\gamma]\right|^{2} \mathrm{~d} s=\frac{2}{\pi} \mathcal{W}(S(\gamma))
$$

where $\kappa_{\mathbb{H}}[\gamma]$ denotes the hyperbolic curvature of $\gamma$ and $S(\gamma)$ denotes the immersion that arises from revolution of $\gamma$ around the $x_{1}$-axis. This and (2) yield that

$$
\int_{\mathbb{S}^{1}}\left|\kappa_{\mathbb{H}}[\gamma]\right|^{2} \mathrm{~d} s<16 \text { implies that } \gamma \text { is an embedding. }
$$

The threshold of 16 is also sharp for this implication, cf. [MS20, Corollary 6.4]. Notice that this does not immediately follow from the sharpness of the inequality in [LY82. In $\mathbb{S}^{2}$ the elastic energy of curves $\gamma \in C^{\infty}\left(\mathbb{S}^{1} ; \mathbb{S}^{2}\right)$ given by

$$
\gamma \mapsto \int_{\mathbb{S}^{1}}\left|\kappa_{\mathbb{S}^{2}}[\gamma]\right|^{2} \mathrm{~d} s
$$

is not an embeddedness-ensuring quantity since any two-fold cover of a closed geodesic in $\mathbb{S}^{2}$ is non-embedded and has vanishing energy.

It would be interesting to investigate whether Theorem 1.2 generalizes to curves in $\mathbb{R}^{3}$, see Remark 5.14 for some ideas in this context.

\section{Notational preliminaries}

In the following, we will view the 1 -sphere as $\mathbb{S}^{1}=[0,1] / \sim$, where $\sim$ denotes the equivalence relation that identifies $0 \sim 1$ and all other points only with themselves. Equivalently, $\mathbb{S}^{1} \cong \mathbb{R} / \mathbb{Z}$. Consequently, an interval $[a, b] \subset \mathbb{S}^{1}$ with $a<b$ has to be understood with respect to this equivalence relation, i.e. $[a, b]=\left\{[x]_{\sim}: x \in[a, b]\right\}$. For the sake of simplicity of notation, we define the interval $[b, a]:=[b, 1] \cup[0, a]$ for $a, b \in[0,1]$ with $a<b$. In the same fashion, the open and half-open intervals are defined. 
Definition 2.1. We define for $k, \ell \in \mathbb{N}, p \in[1, \infty]$ the Sobolev space $W^{k, p}\left(\mathbb{S}^{1} ; \mathbb{R}^{\ell}\right)$ as

$$
W^{k, p}\left(\mathbb{S}^{1} ; \mathbb{R}^{\ell}\right):=\left\{u \in W^{k, p}\left((0,1) ; \mathbb{R}^{\ell}\right) \mid u^{(m)}(0)=u^{(m)}(1) \forall m=1, \ldots, k-1\right\},
$$

where $u^{(m)}$ denotes the continuous representative of the $m$-th weak derivative. Moreover, we denote by $W_{I m m}^{k, p}\left(\mathbb{S}^{1} ; \mathbb{R}^{\ell}\right)$ the set of $W^{k, p}$-immersions.

Remark 2.2. It can be seen that this definition coincides with the general definition of Sobolev spaces on manifolds, cf. [Heb96, Definition 2.1]. This is why we can also use general results about these spaces and also talk about Sobolev spaces on open subsets of $\mathbb{S}^{1}$. We will refer to curves in $C^{k}\left(\mathbb{S}^{1} ; \mathbb{R}^{2}\right)$ as $C^{k}$-closed, which is also due to the fact that

$$
C^{k}\left(\mathbb{S}^{1} ; \mathbb{R}^{2}\right)=\left\{u \in C^{k}\left([0,1] ; \mathbb{R}^{2}\right) \mid u^{(m)}(0)=u^{(m)}(1) \forall m=0, \ldots, k\right\} .
$$

In particular, each curve $W^{k, p}\left(\mathbb{S}^{1} ; \mathbb{R}^{2}\right)$ is $C^{k-1}$-closed. Observe also that each curve in $C^{k}\left(\mathbb{S}^{1} ; \mathbb{R}^{2}\right)$ possesses an extension to a 1 -periodic curve in $C^{k}\left(\mathbb{R}, \mathbb{R}^{2}\right)$.

Remark 2.3. Another noticable property of $W^{k, p}\left(\mathbb{S}^{1}\right)$ is glueing, i.e. if $u \in W^{k, p}((a, b))$ and $v \in W^{k, p}((b, a))$ are such that $u^{(m)}(a)=v^{(m)}(a)$ and $u^{(m)}(b)=v^{(m)}(b)$ for all $m=0, \ldots, k-1$ then

$$
w(x):= \begin{cases}u(x) & x \in(a, b) \\ v(x) & x \in(b, a)\end{cases}
$$

lies in $W^{k, p}\left(\mathbb{S}^{1}\right)$.

We now review some basic geometric definitions of planar curves. For an immersion $\gamma: \mathbb{S}^{1} \rightarrow \mathbb{R}^{2}$ we write $\gamma(x)=\left(\gamma_{1}(x), \gamma_{2}(x)\right), x \in \mathbb{S}^{1}$ for the components and $\gamma^{\prime}=\partial_{x} \gamma$ for the derivative. Moreover, we write $\kappa:=\left|\gamma^{\prime}\right|^{-3} \operatorname{det}\left(\gamma^{\prime}, \gamma^{\prime \prime}\right)$ for its (signed) curvature. Another important geometric object is the arc-length derivative, denoted by $\partial_{s}=\left|\gamma^{\prime}\right|^{-1} \partial_{x}$ and the arc-length element $\mathrm{d} s:=\left|\gamma^{\prime}\right| \mathrm{d} x$. The curvature vector field is $\vec{\kappa}=\partial_{s}^{2} \gamma=\kappa \vec{n}$, where $\vec{n}$ denotes the unit normal, obtained by rotating $\partial_{s} \gamma$ counterclockwise by $\frac{\pi}{2}$.

\section{A non-existence result for the total curvature}

In this section, we will prove Theorem 1.1 and show why it implies that there is no non-trivial generalization of (2) involving the total curvature.

Proof of Theorem 1.1. By Fenchel's theorem, cf. Theorem A.1, we have $\mathcal{K}(\gamma) \geq 2 \pi$ for all $\gamma \in C^{2}\left(\mathbb{S}^{1} ; \mathbb{R}^{2}\right)$. Consequently

$$
\inf \left\{\mathcal{K}(\gamma) \mid \gamma \in C^{2}\left(\mathbb{S}^{1} ; \mathbb{R}^{2}\right) \text { non-embedded immersion }\right\} \geq 2 \pi .
$$

To prove equality, we take some angle $\beta \in\left(0, \frac{\pi}{2}\right)$. First, take a segment $S_{\beta}$ of a circle of radius 1 of length $2 \pi-2 \beta$ and place it symmetrically with respect to the $x_{1}$-axis. Extend the segment by the tangent lines at its endpoints. For $\beta \in\left(0, \frac{\pi}{2}\right)$, they will intersect in a point on the $x_{1}$-axis. Reflecting everything with respect to that point gives a closed curve $\gamma$ which is not embedded, cf. Figure 1a. 


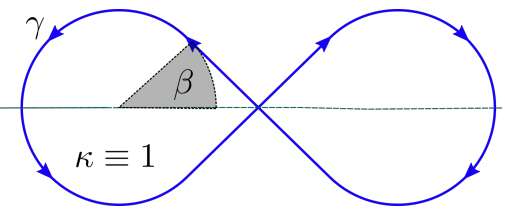

(a) The construction with $\beta=\frac{\pi}{4}$

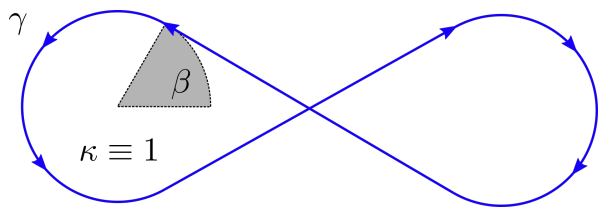

(b) The curve becomes longer as $\beta \nearrow \frac{\pi}{2}$

Figure 1.: A non-embedded $W^{2,2}$-curve with approximate total curvature $2 \pi$ as $\beta \nearrow \frac{\pi}{2}$.

By the glueing property in Remark 2.3 , we have $\gamma \in W^{2,2}\left(\mathbb{S}^{1} ; \mathbb{R}^{2}\right)$. In order to compute $\mathcal{K}(\gamma)$ we first note that $|\kappa| \equiv 1$ on the left circle segment, whereas $\kappa \equiv 0$ on the straight line. Thus by symmetry

$$
\mathcal{K}(\gamma)=2 \int_{S_{\beta}} \mathrm{d} s=2(2 \pi-2 \beta)=4 \pi-4 \beta \searrow 2 \pi, \text { as } \beta \nearrow \frac{\pi}{2} .
$$

Using the characterization of the equality in Fenchel's theorem (Theorem A.1), we may conclude that the infimum in (4) is not attained by a non-embedded immersion.

Theorem 1.2 shows that $\mathcal{K}$ cannot distinguish between embedded and non-embedded immersions, since the least possible energy among all closed curves can be approximated by non-embedded ones. This justifies that (2) has no non-trival generalization to the total curvature.

\section{The variational problem and existence of a minimizer}

In order to prove Theorem 1.2 , we wish to minimize the functional $\mathcal{E}(\gamma) \mathcal{L}(\gamma)$ among all curves $\gamma \in W_{I m m}^{2,2}\left(\mathbb{S}^{1} ; \mathbb{R}^{2}\right)$ which are not embeddings.

As a first step, we want to characterize embeddedness in a way that is useful for variational discussions.

Lemma 4.1. A curve $\gamma \in W_{\text {Imm }}^{2,2}\left(\mathbb{S}^{1}, \mathbb{R}^{2}\right)$ is an embedding, if and only if $\gamma$ is injective.

Proof. See for instance [Lee03, Proposition 5.4].

This implies the following very useful characterization of embeddedness.

Proposition 4.2 (Characterization of Embeddedness). Let $\gamma \in W^{2,2}\left(\mathbb{S}^{1} ; \mathbb{R}^{2}\right)$ be an immersion. Then $\gamma$ is embedded if and only if $A[\gamma]:=\inf _{x \neq y} \frac{|\gamma(x)-\gamma(y)|}{|x-y|}>0$.

Proof. Suppose $A[\gamma]>0$. Then, we have $|\gamma(x)-\gamma(y)| \geq A[\gamma]|x-y|>0$ for $x \neq y \in \mathbb{S}^{1}$. Thus $\gamma$ is injective, hence an embedding by Lemma 4.1 .

Conversely, suppose $\gamma$ is an embedding and $A[\gamma]=0$. Then, there exist $x_{n} \neq y_{n}$ such that $\frac{\left|\gamma\left(x_{n}\right)-\gamma\left(y_{n}\right)\right|}{\left|x_{n}-y_{n}\right|} \rightarrow 0$. Passing to a subsequence, we have $x_{n} \rightarrow x, y_{n} \rightarrow y$ for $x, y \in \mathbb{S}^{1}$ by compactness. If $x \neq y$, we have

$$
0=\lim _{n \rightarrow \infty} \frac{\left|\gamma\left(x_{n}\right)-\gamma\left(y_{n}\right)\right|}{\left|x_{n}-y_{n}\right|}=\frac{|\gamma(x)-\gamma(y)|}{|x-y|},
$$


hence $\gamma(x)=\gamma(y)$. This is a contradiction to the embeddedness of $\gamma$, cf. Lemma 4.1 . Hence $x=y$ in $\mathbb{S}^{1}$ and for $i=1,2$ we have

$$
\gamma_{i}\left(x_{n}\right)-\gamma_{i}\left(y_{n}\right)=\gamma_{i}^{\prime}\left(\xi_{i, n}\right)\left(x_{n}-y_{n}\right)
$$

for some $\xi_{i, n} \in \mathbb{S}^{1}$ between $x_{n}$ and $y_{n}$. Dividing by $x_{n}-y_{n}$ and using the assumption, we find

$$
\gamma_{i}^{\prime}(x)=\lim _{n \rightarrow \infty} \gamma_{i}^{\prime}\left(\xi_{i}, n\right)=\lim _{n \rightarrow \infty} \frac{\gamma_{i}\left(x_{n}\right)-\gamma_{i}\left(y_{n}\right)}{x_{n}-y_{n}}=0,
$$

for $i=1,2$, a contradiction to $\gamma$ being an immersion.

An important consequence is the following lemma.

Lemma 4.3. The set of $C^{1}$-embeddings is an open subset of $C^{1}\left(\mathbb{S}^{1} ; \mathbb{R}^{2}\right)$.

Proof. Suppose $\gamma \in C^{1}\left(\mathbb{S}^{1} ; \mathbb{R}^{2}\right)$ is an embedding. We claim that for $\varepsilon>0$ small enough any $\tilde{\gamma} \in C^{1}\left(\mathbb{S}^{1} ; \mathbb{R}^{2}\right)$ with $\|\tilde{\gamma}-\gamma\|_{C^{1}}<\varepsilon$ is an embedding. By Proposition 4.2 , it suffices to show $A[\tilde{\gamma}]>0$, since $\tilde{\gamma}$ is clearly an immersion for $\varepsilon>0$ small enough. We have for $x \neq y \in \mathbb{S}^{1}$

$$
\begin{aligned}
\frac{|\tilde{\gamma}(x)-\tilde{\gamma}(y)|}{|x-y|} & \geq \frac{|\gamma(x)-\gamma(y)|-\mid \tilde{\gamma}(x)-\gamma(x)-(\tilde{\gamma}(y))-\gamma(y)) \mid}{|x-y|} \\
& \geq A[\gamma]-\sup _{x \neq y} \frac{\mid \tilde{\gamma}(x)-\gamma(x)-(\tilde{\gamma}(y))-\gamma(y)) \mid}{|x-y|} \\
& \geq A[\gamma]-\|\tilde{\gamma}-\gamma\|_{C^{1}} \\
& \geq A[\gamma]-\varepsilon>0
\end{aligned}
$$

if $\varepsilon>0$ is small enough.

Remark 4.4. In the proofs of Proposition 4.2 and Lemma 4.3, we did not really use the specific structure of $\mathbb{S}^{1}$. In particular, the statements of Proposition 4.2 and Lemma 4.3 remain true if one replaces $\mathbb{S}^{1}$ by any compact interval $[a, b] \subset \mathbb{R}$.

We now consider a minimization problem, cf. (3). Define the set

$$
\mathcal{A}:=\left\{\gamma \in W^{2,2}\left(\mathbb{S}^{1} ; \mathbb{R}^{2}\right) \mid \gamma \text { is a non-injective immersion }\right\} \subset W^{2,2}\left(\mathbb{S}^{1} ; \mathbb{R}^{2}\right) .
$$

Theorem 4.5 (Existence of a Minimizer). There exists $\bar{\gamma} \in \mathcal{A}$ with

$$
\mathcal{E}(\bar{\gamma}) \mathcal{L}(\bar{\gamma})=\inf _{\gamma \in \mathcal{A}} \mathcal{E}(\gamma) \mathcal{L}(\gamma)>0
$$

Proof. We consider the set $\tilde{\mathcal{A}}:=\{\gamma \in \mathcal{A} \mid \mathcal{L}(\gamma)=1\} \neq \emptyset$. As a first step, we show that there exist $\bar{\gamma} \in \tilde{\mathcal{A}}$ with

$$
\mathcal{E}(\bar{\gamma})=\inf _{\gamma \in \tilde{\mathcal{A}}} \mathcal{E}(\gamma)
$$


Let $\left(\gamma^{(n)}\right)_{n \in \mathbb{N}}$ be a minimizing sequence for (6). Without loss of generality, we may assume $\gamma^{(n)}$ to be unit speed parametrized for all $n \in \mathbb{N}$, cf. Lemma A.6. By reflexivity and the compactness of the embedding $W^{2,2}\left(\mathbb{S}^{1} ; \mathbb{R}^{2}\right) \hookrightarrow C^{1}\left(\mathbb{S}^{1} ; \mathbb{R}^{2}\right)$, we have $\gamma^{(n)} \rightarrow \bar{\gamma}$ in $W^{2,2}\left(\mathbb{S}^{1} ; \mathbb{R}^{2}\right)$ and $\gamma^{(n)} \rightarrow \bar{\gamma}$ in $C^{1}\left(\mathbb{S}^{1} ; \mathbb{R}^{2}\right)$ for some $\bar{\gamma} \in W^{2,2}\left(\mathbb{S}^{1} ; \mathbb{R}^{2}\right)$, passing to a subsequence. We have $\left|\gamma_{n}^{\prime}(x)\right|=1$ for all $x \in \mathbb{S}^{1}, n \in \mathbb{N}$, thus $\bar{\gamma}$ is parametrized with unit speed and $\mathcal{L}(\bar{\gamma})=1$. Moreover, by Lemma $4.3, \bar{\gamma}$ cannot be an embedding as $\gamma^{(n)} \rightarrow \bar{\gamma}$ in $C^{1}\left(\mathbb{S}^{1} ; \mathbb{R}^{2}\right)$. Consequently we have $\bar{\gamma} \in \mathcal{\mathcal { A }}$ by Lemma 4.1

For unit speed curves $\gamma \in W^{2,2}\left(\mathbb{S}^{1} ; \mathbb{R}^{2}\right)$, the elastic energy is given by

$$
\mathcal{E}(\gamma)=\int_{\mathbb{S}^{1}}\left|\gamma^{\prime \prime}(x)\right|^{2} \mathrm{~d} x
$$

Since the $L^{2}\left(\mathbb{S}^{1} ; \mathbb{R}^{2}\right)$-norm is weakly lower semicontinuous, we conclude

$$
\mathcal{E}(\bar{\gamma}) \leq \liminf _{n \rightarrow \infty} \mathcal{E}\left(\gamma_{n}\right)=\inf _{\gamma \in \tilde{\mathcal{A}}} \mathcal{E}(\gamma)
$$

so $\bar{\gamma}$ is a minimizer. Since $\mathbb{R}^{2}$ does not allow for closed geodesics one infers that $\mathcal{E}(\bar{\gamma})>0$. Now, if $\gamma \in W^{2,2}\left(\mathbb{S}^{1} ; \mathbb{R}^{2}\right)$ is an arbitrary immersion, we may consider the rescaled curve $\tilde{\gamma}:=\frac{1}{\mathcal{L}(\gamma)} \gamma$, so $\tilde{\gamma} \in \tilde{\mathcal{A}}$. Note that $\gamma$ is an embedding if and only if $\tilde{\gamma}$ is an embedding. Consequently, we have

$$
\mathcal{E}(\gamma) \mathcal{L}(\gamma)=\mathcal{E}(\tilde{\gamma}) \geq \mathcal{E}(\bar{\gamma})=\mathcal{E}(\bar{\gamma}) \mathcal{L}(\bar{\gamma})
$$

and hence $\inf _{\gamma \in \mathcal{A}} \mathcal{E}(\gamma) \mathcal{L}(\gamma)=\mathcal{E}(\bar{\gamma}) \mathcal{L}(\bar{\gamma})$.

\section{The Euler-Lagrange equation}

In this section, we will study the properties of a minimizer $\bar{\gamma}$ from Theorem 4.5 in order to prove our main theorem. The most important property we will derive is that $\bar{\gamma}$ is a constrained elastica, i.e. $\bar{\gamma} \in C^{\infty}\left(\mathbb{S}^{1} ; \mathbb{R}^{2}\right)$ and

$$
\partial_{s}^{2} \kappa+\frac{1}{2} \kappa^{3}-\lambda \kappa=0 \quad \text { for some } \lambda \in \mathbb{R} .
$$

Solutions of the constrained elastica equation have been classified in previous works, eg. by [LS84c, DHMV08. What one needs to show for this is that $\bar{\gamma}$ satisfies the EulerLagrange equation, i.e. for all $\phi \in C^{\infty}\left(\mathbb{S}^{1} ; \mathbb{R}^{2}\right)$ one has

$$
\mathcal{L}(\bar{\gamma}) D \mathcal{E}(\bar{\gamma})(\phi)+\mathcal{E}(\bar{\gamma}) D \mathcal{L}(\bar{\gamma})(\phi)=0 .
$$

Indeed, as one can see following the lines of [EG19, Section 5], (8) implies that $\bar{\gamma}$ is smooth and (7) holds. If $\bar{\gamma}$ is an interior point of $\mathcal{A}$ then (8) follows from the fact that for all $\phi \in C^{\infty}\left(\mathbb{S}^{1} ; \mathbb{R}^{2}\right)$ one has

$$
0=\left.\frac{\mathrm{d}}{\mathrm{d} \varepsilon}\right|_{\varepsilon=0} \mathcal{E}(\bar{\gamma}+\varepsilon \phi) \mathcal{L}(\bar{\gamma}+\varepsilon \phi),
$$




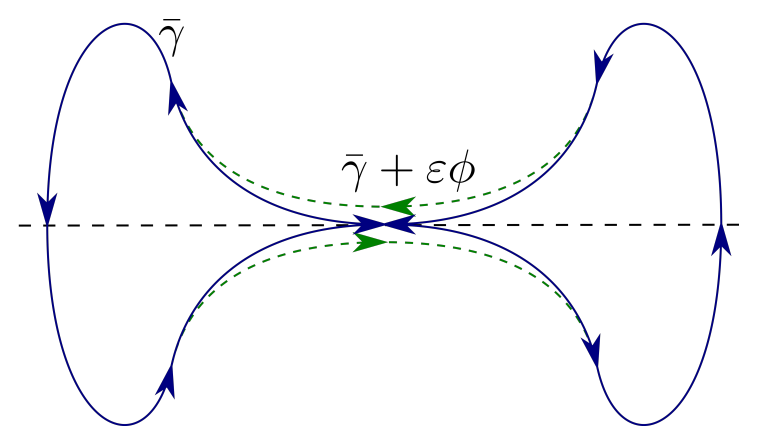

Figure 2.: A tangential self intersection vanishing under small perturbations.

since $\left(-\varepsilon_{0}, \varepsilon_{0}\right) \ni \varepsilon \mapsto \mathcal{E}(\bar{\gamma}+\varepsilon \phi) \mathcal{L}(\bar{\gamma}+\varepsilon \phi)$ attains a minimum at $\varepsilon=0$. The problem is that we do not know to begin with whether the infimum is attained at an interior point of $\mathcal{A}$. The goal of this section is to prove exactly this.

We remark that $\mathcal{A} \subset W^{2,2}\left(\mathbb{S}^{1} ; \mathbb{R}^{2}\right)$ is not open. Indeed, like in Figure 2 it is possible to leave the set $\mathcal{A}$ by a variation that eliminates self-intersections. To show that each minimizer is an interior point we need to examine these self-intersections, denoted by

$$
S[\gamma]:=\left\{p \in \mathbb{R}^{2}: \exists x_{1} \neq x_{2} \text { s.t. } \gamma\left(x_{1}\right)=\gamma\left(x_{2}\right)=p\right\} .
$$

We also define the multiplicity of $p \in S[\gamma]$ to be $\operatorname{mult}[\gamma](p):=\mathcal{H}^{0}\left(\gamma^{-1}(\{p\})\right)$ where $\mathcal{H}^{0}$ denotes the counting measure. Moreover we have to pay special attention to the tangential self-intersections given by

$$
S_{\text {tan }}[\gamma]:=\left\{p \in \mathbb{R}^{2}: \exists x_{1} \neq x_{2} \text { s.t. } \gamma\left(x_{1}\right)=\gamma\left(x_{2}\right)=p, \operatorname{det}\left(\gamma^{\prime}\left(x_{1}\right), \gamma^{\prime}\left(x_{2}\right)\right)=0\right\} .
$$

Before we can proceed with the proof we need some preparations that exclude certain configurations.

\subsection{Some facts about constrained elasticae}

In this section, we will discuss properties of closed elasticae. We remark that at this point we do not know whether the minimizer $\bar{\gamma}$ is a constrained elastica. However, the analysis of the energy and the self-intersection properties of closed elasticae will play a crucial role in proving Theorem 1.2 later. More precisely, we will be able to exclude self-intersection properties of minimizers $\bar{\gamma}$ once we can exclude them for non-embedded constrained elasticae. Define

$$
\mathcal{B}:=\left\{\gamma \in C^{\infty}\left(\mathbb{S}^{1} ; \mathbb{R}^{2}\right): \gamma \text { is a non-embedded constrained elastica }\right\} \subset \mathcal{A} .
$$

Throughout this section we will use the elliptic functions defined in Appendix B. We next define one special elastica, which will be important for our considerations.

Definition 5.1 (The Elastic Figure Eight). Let $m^{*} \in(0,1)$ be the unique root of the map $(0,1) \ni m \mapsto 2 E(m)-K(m)$, see Lemma B.4. We define the one-fold cover of the 
elastic figure eight by

$$
\gamma^{*}:\left[0,4 K\left(m^{*}\right)\right] \rightarrow \mathbb{R}^{2}, \quad \gamma^{*}(s):=\left(\begin{array}{c}
2 E\left(\operatorname{am}\left(s, m^{*}\right), m^{*}\right)-s \\
-2 \sqrt{m^{*}} \operatorname{cn}\left(s, m^{*}\right)
\end{array}\right) .
$$

Remark 5.2. The numerical value of $m^{*}$ is $m^{*} \simeq 0.8261$. This is not needed in the sequel, since all the computations we provide are analytical. However, it enables us to approximate the value of $c^{*}$.

Remark 5.3. We will also look at another parametrization of the figure eight that is easier for computations e.g. to compute self-intersections. For this observe that by (11) and Appendix B

$$
\gamma^{*}\left(F\left(x, m^{*}\right)\right)=\left(\begin{array}{c}
2 E\left(x, m^{*}\right)-F\left(x, m^{*}\right) \\
-2 \sqrt{m^{*}} \cos (x)
\end{array}\right) .
$$

and note that $F\left(\cdot, m^{*}\right)$ is strictly monotone with $F\left(0, m^{*}\right)=0$ and $F\left(2 \pi, m^{*}\right)=4 K\left(m^{*}\right)$. Hence

$$
\tilde{\gamma}^{*}:[0,2 \pi] \rightarrow \mathbb{R}^{2}, \quad \tilde{\gamma}^{*}(x):=\left(\begin{array}{c}
2 E\left(x, m^{*}\right)-F\left(x, m^{*}\right) \\
-2 \sqrt{m^{*}} \cos (x)
\end{array}\right)
$$

is also a parametrization of the figure eight.

We show next that the elastic figure eight is smoothly closed and minimizes our functional in $\mathcal{B}$. It will turn out later that it is actually also a minimizer in $\mathcal{A}$.

Lemma 5.4 (Characterization of Closed Elasticae). The only closed constrained elasticae are (possibly rescaled, rotated, translated and reparametrized versions of) multi-fold coverings of circles and multi-fold coverings of the figure eight. All of these elasticae are not embedded except for the one-fold covering of the circle. Moreover,

$$
\inf _{\gamma \in \mathcal{B}} \mathcal{E}(\gamma) \mathcal{L}(\gamma)=\mathcal{E}\left(\gamma^{*}\right) \mathcal{L}\left(\gamma^{*}\right)
$$

with $\gamma^{*}$ as in Definition 5.1. Equality holds if and only if $\gamma$ is a rescaled, translated and rotated reparametrization of $\gamma^{*}$.

Proof. First we show the assertion that the only closed elasticae are given by the figure eight and the circle. By Proposition B.8 there are - up to scaling, isometries in $\mathbb{R}^{2}$, and reparametrization - only five different types of elasticae which we all examine seperately for closedness.

Type 1: Linear elasticae. Since lines are not closed they cannot generate closed elasticae.

Type 2: Wavelike elasticae. For closedness it is necessary that both components of $\gamma$ are periodic with the same period $L$, cf. Remark 2.2. The period itself does not matter since we can always reparametrize the curve. In the wavelike case we have by Proposition B.8

$$
\gamma_{1}(s)=2 E(\operatorname{am}(s, m), m)-s \quad \gamma_{2}(s)=-2 \sqrt{m} \operatorname{cn}(s, m) .
$$


By Proposition B.3 all periods $L$ of $\gamma_{2}$ are given by

$$
L \in\{4 l K(m): l \in \mathbb{N}\} .
$$

We investigate with the aid of Proposition B.3 for which values of $m$ one of these periods is also a period of $\gamma_{1}$. For $l \in \mathbb{N}$ we compute

$$
\begin{aligned}
\gamma_{1}(s+4 l K(m)) & =2 E(\operatorname{am}(s, m)+2 l \pi, m)-s-4 l K(m) \\
& =2 E(\operatorname{am}(s, m), m)+8 l E(m)-s-4 l K(m) \\
& =\gamma_{1}(s)+4 l(2 E(m)-K(m)) .
\end{aligned}
$$

Hence $\gamma_{1}$ and $\gamma_{2}$ share a period if and only if $2 E(m)-K(m)=0$. In this case $4 K(m)$ is already a joint period of $\gamma_{1}$ and $\gamma_{2}$, hence a period of $\gamma$. By Lemma B.4. $2 E(m)-K(m)$ has only one zero $m^{*} \in(0,1)$, which by Definition 5.1 yields exactly the elastic figure eight.

Type 3: Borderline elastica. Can not be periodic since the second component has no real period.

Type 4: Orbitlike elasticae. We proceed similar to the wavelike case. Recall that by Proposition B.8

$$
\gamma_{1}(s)=\frac{2}{m} E(\operatorname{am}(s, m), m)+\left(1-\frac{2}{m}\right) s, \quad \gamma_{2}(s)=-\frac{2}{m} \operatorname{dn}(s, m) .
$$

By Proposition B.3 all periods $L$ of $\gamma_{2}$ are given by

$$
L \in\{2 l K(m): l \in \mathbb{N}\}
$$

Next we look at the behavior of $\gamma_{1}$, which we can characterize by Proposition B.3 to be

$$
\gamma_{1}(s+2 l K(m))=\gamma_{1}(s)+l \frac{4 E(m)-4 K(m)+2 m K(m)}{m} .
$$

Hence $\gamma_{1}$ and $\gamma_{2}$ share a period if and only if $4 E(m)-4 K(m)+2 m K(m)=0$, which is impossible because of Lemma B.5. Hence there do not exist closed orbitlike elasticae.

Type 5: Circular elasticae. Circles are trivially closed. Their period is given by $2 \pi$ when using the standard arclength parametrization $x \mapsto(\cos (x), \sin (x))$.

Summarizing our findings for all the types we obtain that the only closed elasticae are circles and the figure eight as well as multiple coverings of these. We have also found their periods. Next we show that the one-fold cover of the figure eight is not injective, so that it is admissible for the minimization problem in the statement. For this let $\gamma^{*}=\left(\gamma_{1}^{*}, \gamma_{2}^{*}\right)$ be the figure eight. A crucial oberservation is that the first component $\gamma_{1}^{*}$ of $\gamma^{*}$ is already $2 K\left(m^{*}\right)$-periodic as one could compute with the same techniques as in (12). Note that

$$
\gamma^{*}\left(K\left(m^{*}\right)\right)=\left(\begin{array}{c}
2 E\left(m^{*}\right)-K\left(m^{*}\right) \\
-2 \sqrt{m^{*}} \cos \left(\frac{\pi}{2}\right)
\end{array}\right)=\left(\begin{array}{l}
0 \\
0
\end{array}\right)
$$


and by the $2 K\left(m^{*}\right)$ periodicity of $\gamma_{1}^{*}$ we obtain

$$
\gamma^{*}\left(3 K\left(m^{*}\right)\right)=\left(\begin{array}{c}
2 E\left(m^{*}\right)-K\left(m^{*}\right) \\
-2 \sqrt{m^{*}} \cos \left(3 \frac{\pi}{2}\right)
\end{array}\right)=\left(\begin{array}{l}
0 \\
0
\end{array}\right) .
$$

Hence $\gamma^{*}\left(K\left(m^{*}\right)\right)=\gamma\left(3 K\left(m^{*}\right)\right)$. Next we show that the figure eight is minimizing. For this we compare the energy of the one-fold cover of the figure eight to the energy of the doubly-covered circle. The energy of the doubly covered circle $\gamma_{d c c}$ is given by

$$
\mathcal{E}\left(\gamma_{d c c}\right) \mathcal{L}\left(\gamma_{d c c}\right)=4 \pi \cdot 4 \pi=16 \pi^{2} .
$$

For the energy of the figure eight note that $\mathcal{L}\left(\gamma^{*}\right)=4 K\left(m^{*}\right)$ as $\gamma^{*}$ is arc-length parametrized by the construction in Appendix B. Now

$$
\begin{aligned}
\mathcal{E}\left(\gamma^{*}\right) & =\int_{0}^{4 K\left(m^{*}\right)} 4 m^{*} \operatorname{cn}^{2}\left(s, m^{*}\right) \mathrm{d} s=4 m^{*} \int_{0}^{2 \pi} \frac{\cos ^{2}(\theta)}{\sqrt{1-m^{*} \sin ^{2}(\theta)}} \mathrm{d} \theta \\
& =16\left[\left(m^{*}-1\right) K\left(m^{*}\right)+E\left(m^{*}\right)\right] .
\end{aligned}
$$

Hence

$$
\mathcal{E}\left(\gamma^{*}\right) \mathcal{L}\left(\gamma^{*}\right)=64\left(E\left(m^{*}\right) K\left(m^{*}\right)+\left(m^{*}-1\right) K\left(m^{*}\right)^{2}\right) .
$$

Using that by Definition $5.12 E\left(m^{*}\right)=K\left(m^{*}\right)$ we have

$$
\mathcal{E}\left(\gamma^{*}\right) \mathcal{L}\left(\gamma^{*}\right)=64\left(4 m^{*}-2\right) E\left(m^{*}\right)^{2} .
$$

We show that this quantity is smaller than $16 \pi^{2}$. For this we use that by Lemma B.7 one has

$$
64\left(4 m^{*}-2\right) E\left(m^{*}\right)^{2} \leq 16\left(2 m^{*}-1\right)\left(2-m^{*}\right) \pi^{2} .
$$

With standard arguments it can be shown that $g(z):=(2 z-1)(2-z)$ is strictly monotone on $(0,1]$ and $g(1)=1$ which makes $\left(2 m^{*}-1\right)\left(2-m^{*}\right)=g\left(m^{*}\right)<1$. Therefore, by (14) and (15)

$$
\mathcal{E}\left(\gamma^{*}\right) \mathcal{L}\left(\gamma^{*}\right)=64\left(4 m^{*}-2\right) E\left(m^{*}\right)^{2}<16 \pi^{2},
$$

which implies by 13 that

$$
\mathcal{E}\left(\gamma^{*}\right) \mathcal{L}\left(\gamma^{*}\right)<\mathcal{E}\left(\gamma_{d c c}\right) \mathcal{L}\left(\gamma_{d c c}\right) .
$$

Remark 5.5. From (16) and Remark 5.2 one may compute $c^{*} \simeq 112.439609741$.

Lemma 5.6. Let $\gamma^{*}$ be as in Definition 5.1. Then $S\left[\gamma^{*}\right]=\{0\}$ with mult $\left[\gamma^{*}\right](0)=1$. Moreover, $S_{\text {tan }}\left[\gamma^{*}\right]=\emptyset$.

Proof. Note that the assertion is not affected by reparametrization. We will work with reparametrizations in the sequel. More exactly, we work with the parametrization $\tilde{\gamma}^{*}$ from Remark 5.3 , given by

$$
\tilde{\gamma}^{*}:[0,2 \pi] \rightarrow \mathbb{R}^{2}, \quad\left(\begin{array}{c}
\tilde{\gamma}_{1}^{*}(x) \\
\tilde{\gamma}_{2}^{*}(x)
\end{array}\right)=\left(\begin{array}{c}
2 E\left(x, m^{*}\right)-F\left(x, m^{*}\right) \\
-2 \sqrt{m^{*}} \cos (x)
\end{array}\right) .
$$


Now let $x_{1}, x_{2} \in[0,2 \pi)$ be such that $\tilde{\gamma}^{*}\left(x_{1}\right)=\tilde{\gamma}^{*}\left(x_{2}\right)$ and, without loss of generality, $x_{1}<x_{2}$. Note that $\tilde{\gamma}_{2}^{*}\left(x_{1}\right)=\tilde{\gamma}_{2}^{*}\left(x_{2}\right)$ yields that $\cos \left(x_{1}\right)=\cos \left(x_{2}\right)$ which implies - since $x_{1}, x_{2} \in[0,2 \pi)$ that $x_{1}=2 \pi-x_{2}$. We infer that

$$
\begin{aligned}
\tilde{\gamma}_{1}^{*}\left(x_{1}\right) & =\tilde{\gamma}_{1}^{*}\left(x_{2}\right)=\tilde{\gamma}_{1}^{*}\left(2 \pi-x_{1}\right)=2 E\left(2 \pi-x_{1}, m^{*}\right)-F\left(2 \pi-x_{1}, m^{*}\right) \\
& =2 E\left(-x_{1}, m^{*}\right)-F\left(-x_{1}, m^{*}\right)+\left(2 E\left(m^{*}\right)-K\left(m^{*}\right)\right) \\
& =2 E\left(-x_{1}, m^{*}\right)-F\left(-x_{1}, m^{*}\right)=-\left(2 E\left(x_{1}, m\right)-F\left(x_{1}, m\right)\right)=-\tilde{\gamma}_{1}^{*}\left(x_{1}\right) .
\end{aligned}
$$

Hence $\tilde{\gamma}_{1}^{*}\left(x_{1}\right)=0$ and therefore also $\tilde{\gamma}_{1}^{*}\left(x_{2}\right)=0$. This means that $x_{1}$ and $x_{2}$ are solutions of

$$
2 E\left(x, m^{*}\right)-F\left(x, m^{*}\right)=0
$$

By Lemma B.6 this implies that

$$
x_{1}, x_{2} \in\left\{0, \frac{\pi}{2}, \pi, \frac{3 \pi}{2}\right\} .
$$

Since also $x_{1}=2 \pi-x_{2}$ this leaves the only possibility of $x_{1}=\frac{\pi}{2}, x_{2}=\frac{3 \pi}{2}$. We obtain that the only self intersection point occurs at $\tilde{\gamma}^{*}\left(\frac{\pi}{2}\right)=\tilde{\gamma}^{*}\left(\frac{3 \pi}{2}\right)=(0,0)^{T}$. Consequently, $S\left[\tilde{\gamma}^{*}\right]=\left\{(0,0)^{T}\right\}$ with mult $\left[\tilde{\gamma}^{*}\right]\left((0,0)^{T}\right)=2$. It remains to show that $S_{\tan }\left[\tilde{\gamma}^{*}\right]=\emptyset$, i.e. the self-intersection is not tangential. To do so we compute

$$
\tilde{\gamma}_{1}^{* \prime}(x)=\frac{1-2 m^{*} \sin ^{2}(x)}{\sqrt{1-m^{*} \sin ^{2}(x)}} \quad \quad_{2}^{* \prime}(x)=2 \sqrt{m^{*}} \sin (x)
$$

and therefore we have $\tilde{\gamma}^{* \prime}\left(\frac{\pi}{2}\right)=\left(\frac{1-2 m^{*}}{\sqrt{1-m^{*}}}, 2 \sqrt{m^{*}}\right)^{T}, \tilde{\gamma}^{* \prime}\left(\frac{3 \pi}{2}\right)=\left(\frac{1-2 m^{*}}{\sqrt{1-m^{*}}},-2 \sqrt{m^{*}}\right)^{T}$ and thus

$$
\operatorname{det}\left(\tilde{\gamma}^{* \prime}\left(\frac{\pi}{2}\right), \tilde{\gamma}^{* \prime}\left(\frac{3 \pi}{2}\right)\right)=-4 \frac{\left(1-2 m^{*}\right) \sqrt{m^{*}}}{\sqrt{1-m^{*}}} \neq 0
$$

as $m^{*} \neq \frac{1}{2}$ by Lemma B.4.

\subsection{Self-intersection properties of minimizers}

In this section, we will prove that every minimizer in (5) is a constrained elastica and thus has to be the figure eight by Lemma 5.4, after rescaling, rotation, translation and reparametrization. This is a crucial and non-standard step in proving our main result. First, we examine the number of intersection points and their multiplicities. Then we show that minimizers have no tangential self-intersections. This allows us to conclude that all minimzers are interior points of $\mathcal{A}$, and hence elasticae. The key idea here is comparing the minimizer to the figure eight elastica defined in Definition 5.1.

Since self-intersection points are delicate to examine we will always localize. We say that $\bar{\gamma}$ solves the Euler-Lagrange equation weakly on an open set $U \subset \mathbb{S}^{1}$ if $(8)$ holds for all $\phi \in C_{0}^{\infty}\left(U ; \mathbb{R}^{2}\right)$.

Lemma 5.7. Let $\bar{\gamma} \in \mathcal{A}$ be a minimizer of (5). Suppose that $x \in \mathbb{S}^{1}$ is such that $\bar{\gamma}(x) \notin S[\gamma]$. Then there exists an open neighborhood $U$ of $x$ in $\mathbb{S}^{1}$ such that for all $\phi \in C_{0}^{\infty}\left(U ; \mathbb{R}^{2}\right)$ one has

$$
\mathcal{L}(\bar{\gamma}) D \mathcal{E}(\bar{\gamma})(\phi)+\mathcal{E}(\bar{\gamma}) D \mathcal{L}(\bar{\gamma})(\phi)=0 .
$$


Proof. Fix $x$ as in the statement. Since $\bar{\gamma} \in \mathcal{A}$ we have that $S[\bar{\gamma}] \neq \emptyset$. Hence there exists some $p \in S[\gamma]$ and two distinct values $x^{\prime}, x^{\prime \prime} \in \mathbb{S}^{1}$ such that $\gamma\left(x^{\prime}\right)=\gamma\left(x^{\prime \prime}\right)=p$. Fix one choice of such $p, x^{\prime}, x^{\prime \prime}$. Now set $U:=\mathbb{S}^{1} \backslash\left\{x^{\prime}, x^{\prime \prime}\right\}$ which is open in $\mathbb{S}^{1}$ and contains $x$ as $\gamma(x) \neq p$. Now fix $\phi \in C_{0}^{\infty}\left(U ; \mathbb{R}^{2}\right)$. We prove that $\phi$ satisfies (17). To do so we show that $\bar{\gamma}+\varepsilon \phi \in \mathcal{A}$ for all $\varepsilon \in \mathbb{R}$. As $x^{\prime}, x^{\prime \prime} \notin U$ we obtain that $\phi\left(x^{\prime}\right)=\phi\left(x^{\prime \prime}\right)=0$ and thus for all $\varepsilon \in \mathbb{R}$

$$
(\bar{\gamma}+\varepsilon \phi)\left(x^{\prime}\right)=\bar{\gamma}\left(x^{\prime}\right)=p=\bar{\gamma}\left(x^{\prime \prime}\right)=(\bar{\gamma}+\varepsilon \phi)\left(x^{\prime \prime}\right) .
$$

In particular $\bar{\gamma}+\varepsilon \phi \in \mathcal{A}$ as it has a self-intersection. Equation 17) follows then from the consideration in (9).

Once we have this tool at hand, we can start to study the self-intersections.

Lemma 5.8. Let $\bar{\gamma} \in \mathcal{A}$ be a minimizer of (5). Then $S[\bar{\gamma}]=\left\{p_{0}\right\}$ for some $p_{0} \in \mathbb{R}^{2}$.

Proof. Note first that $S[\bar{\gamma}] \neq \emptyset$ as $\bar{\gamma}$ is not embedded. We proceed showing that $S[\bar{\gamma}]$ is a singleton. Assume that there exist $p_{1}, p_{2} \in S[\bar{\gamma}]$ such that $p_{1} \neq p_{2}$. In particular there exists $\rho>0$ such that $B_{\rho}\left(p_{1}\right) \cap B_{\rho}\left(p_{2}\right)=\emptyset$. We claim that $\bar{\gamma}$ must be a constrained elastica. For this we show that the Euler-Lagrange equation is globally fulfilled. Thus, we have to discuss the behavior at self-intersection points. Fix any $x_{0} \in \mathbb{S}^{1}$ such that $\bar{\gamma}\left(x_{0}\right) \in S[\bar{\gamma}]$. We will derive that $\bar{\gamma}$ solves the Euler-Lagrange equation in a neighborhood of $x_{0}$. We show first that there exists $\delta=\delta\left(x_{0}\right)>0$ such that $\bar{\gamma}\left(\left(x_{0}-\delta, x_{0}+\delta\right)\right)$ has empty intersection with one of $B_{\frac{\rho}{2}}\left(p_{1}\right)$ or $B_{\frac{\rho}{2}}\left(p_{2}\right)$. For this we distinguish two cases, the first one being $\bar{\gamma}\left(x_{0}\right) \notin B_{\rho}\left(p_{1}\right)$. By continuity there exists some $\delta>0$ such that $\left|\bar{\gamma}(x)-\bar{\gamma}\left(x_{0}\right)\right|<\frac{\rho}{2}$ for all $x \in\left(x_{0}-\delta, x_{0}+\delta\right)$ and hence by the triangle inequality $\bar{\gamma}(x) \notin B_{\frac{\rho}{2}}\left(p_{1}\right)$ for all $x \in\left(x_{0}-\delta, x_{0}+\delta\right)$. The second case is $\bar{\gamma}\left(x_{0}\right) \in B_{\rho}\left(p_{1}\right)$, it could as well be equal to $p_{1}$. By the construction of $\rho$ this implies that $\bar{\gamma}\left(x_{0}\right) \notin B_{\rho}\left(p_{2}\right)$ and thus we can repeat the above continuity argument to find that there exists $\delta>0$ such that $\gamma(x) \notin B_{\frac{\rho}{2}}\left(p_{2}\right)$ for all $x \in\left(x_{0}-\delta, x_{0}+\delta\right)$. With this case distinction we have completed the construction of $\delta=\delta\left(x_{0}\right)$. We will without loss of generality assume that $\bar{\gamma}\left(\left(x_{0}-\delta, x_{0}+\delta\right)\right)$ has empty intersection with $B_{\frac{\rho}{2}}\left(p_{2}\right)$, otherwise we switch the roles. We show now that for all $\phi \in C_{0}^{\infty}\left(\left(x_{0}-\delta, x_{0}+\delta\right) ; \mathbb{R}^{2}\right)$ one has

$$
\mathcal{L}(\bar{\gamma}) D \mathcal{E}(\bar{\gamma})(\phi)+\mathcal{E}(\bar{\gamma}) D \mathcal{L}(\bar{\gamma})(\phi)=0 .
$$

To this end we fix $\phi \in C_{0}^{\infty}\left(\left(x_{0}-\delta, x_{0}+\delta\right) ; \mathbb{R}^{2}\right)$. We show that for all $\varepsilon \in \mathbb{R}$ one has $\bar{\gamma}+\varepsilon \phi \in \mathcal{A}$ which implies (18) by minimality of $\bar{\gamma}$. Recall that $p_{2} \in S[\bar{\gamma}]$ and hence there exist $x^{\prime}, x^{\prime \prime} \in \mathbb{S}^{1}$ such that $\bar{\gamma}\left(x^{\prime}\right)=\bar{\gamma}\left(x^{\prime \prime}\right)=p_{2}$. We claim that $x^{\prime}, x^{\prime \prime} \notin\left(x_{0}-\delta, x_{0}+\delta\right)$. Indeed, if we assume e.g that $x^{\prime} \in\left(x_{0}-\delta, x_{0}+\delta\right)$, we obtain by choice of $\delta$ that $p_{2}=\bar{\gamma}\left(x^{\prime}\right) \in \bar{\gamma}\left(\left(x_{0}-\delta, x_{0}+\delta\right)\right)$. This is a contradiction to the fact that $\bar{\gamma}\left(\left(x_{0}-\delta, x_{0}+\delta\right)\right)$ does not intersect $B_{\frac{\rho}{2}}\left(p_{2}\right)$. Similarly one obtains that $x^{\prime \prime} \notin\left(x_{0}-\delta, x_{0}+\delta\right)$. Now we can compute for each $\varepsilon \in \mathbb{R}$ using that $\operatorname{supp} \phi \subset\left(x_{0}-\delta, x_{0}+\delta\right)$

$$
(\bar{\gamma}+\varepsilon \phi)\left(x^{\prime}\right)=\bar{\gamma}\left(x^{\prime}\right)=p_{2}=\bar{\gamma}\left(x^{\prime \prime}\right)=(\bar{\gamma}+\varepsilon \phi)\left(x^{\prime \prime}\right) .
$$

This implies that $\bar{\gamma}+\varepsilon \phi \in \mathcal{A}$ and - as we discussed - also (18). 
Recalling that $x_{0}$ was arbitrary we have shown that for each $x_{0} \in \mathbb{S}^{1}$ such that $x_{0} \in S[\bar{\gamma}]$ there exists an open neighborhood $U_{x_{0}}$ of $x_{0}$ in $\mathbb{S}^{1}$ such that for all $\phi \in C_{0}^{\infty}\left(U_{x_{0}}\right)$ (18) holds true, under the assumption that there exist $p_{1} \neq p_{2} \in S[\bar{\gamma}]$. Since such neighborhood exists also for non-intersection points by Lemma 5.7 we obtain that for each $x \in \mathbb{S}^{1}$ there exists an open neighborhood $U_{x}$ of $x$ in $\mathbb{S}^{1}$ such that the Euler-Lagrange equation (8) holds for all $\phi \in C_{0}^{\infty}\left(U_{x} ; \mathbb{R}^{2}\right)$. Now we can choose a partition of unity of $\mathbb{S}^{1}$ subordinate to the cover $\left\{U_{x}\right\}_{x \in \mathbb{S}^{1}}$ which yields finitely many $\left\{x_{1}, \ldots, x_{N}\right\}$ and nonnegative functions $\eta_{x_{1}}, \ldots, \eta_{x_{N}} \in C^{\infty}\left(\mathbb{S}^{1} ; \mathbb{R}\right)$ such that the support of $\eta_{x_{i}}$ is compactly contained in $U_{x_{i}}$ for all $i=1, \ldots, N$ and

$$
\sum_{i=1}^{N} \eta_{x_{i}}=1
$$

Now fix $\phi \in C^{\infty}\left(\mathbb{S}^{1} ; \mathbb{R}^{2}\right)$. We obtain by 18$)$ and the linearity of the Frechét derivative

$$
\mathcal{L}(\bar{\gamma}) D \mathcal{E}(\bar{\gamma})(\phi)+\mathcal{E}(\bar{\gamma}) D \mathcal{L}(\bar{\gamma})(\phi)=\sum_{i=1}^{N} \mathcal{L}(\bar{\gamma}) D \mathcal{E}(\bar{\gamma})\left(\eta_{x_{i}} \phi\right)+\mathcal{E}(\bar{\gamma}) D \mathcal{L}(\bar{\gamma})\left(\eta_{x_{i}} \phi\right)=0
$$

As discussed after (8), $\bar{\gamma}$ is a constrained elastica. Since $\bar{\gamma}$ minimizes (5), it must then also minimize among non-embedded constrained elasticae. However, the one-fold cover of the figure eight $\gamma^{*}$ is the unique minimizer of $\mathcal{E} \mathcal{L}$ among all constrained elasticae, up to rescaling, rotation and reparametrization, cf. Lemma 5.4. Therefore, $\bar{\gamma}$ has to be a suitably rescaled, rotated and reparametrized version of $\gamma^{*}$. This yields that $S[\bar{\gamma}]$ is a singleton, since $S\left[\gamma^{*}\right]$ is a singleton, a contradiction.

The ideas of the following proofs will be very similar to the preceding one. We assume that a certain configuration exists in $\bar{\gamma}$ and then conlude that $\bar{\gamma}$ has to be a constrained elastica. We then use the classification of those in Section 5.1 to rule out this configuration. The next lemma is in the same spirit.

Lemma 5.9. Let $\bar{\gamma} \in \mathcal{A}$ be a minimizer of (5). Suppose that $p_{0} \in S[\gamma]$. Then $\operatorname{mult}[\bar{\gamma}]\left(p_{0}\right)=2$. In particular there exist exactly two values $x_{1}, x_{2} \in \mathbb{S}^{1}$ such that $x_{1} \neq x_{2}$ and $\gamma\left(x_{1}\right)=\gamma\left(x_{2}\right)$.

Proof. As $S[\bar{\gamma}]$ is a singleton by Lemma 5.8 the last sentence of the claim follows immediately from the multiplicity result. As $p_{0} \in S[\bar{\gamma}]$ we have mult $[\bar{\gamma}]\left(p_{0}\right) \geq 2$. Assume that mult $[\bar{\gamma}]\left(p_{0}\right)>2$. We show that then $\bar{\gamma}$ must be a constrained elastica. To that end, let $x_{0} \in \gamma^{-1}\left(\left\{p_{0}\right\}\right)$ be arbitrary. We claim that there exists a neighborhood $U_{x_{0}}$ on which the Euler Lagrange equation is fulfilled. By the assumption on the multiplicity there exist two distinct values $x^{\prime}, x^{\prime \prime} \in \gamma^{-1}\left(\left\{p_{0}\right\}\right) \backslash\left\{x_{0}\right\}$. Fix a choice of such $x^{\prime}, x^{\prime \prime}$. Choose $\delta=\delta\left(x_{0}\right)>0$ such that $x^{\prime}, x^{\prime \prime} \notin\left(x_{0}-\delta, x_{0}+\delta\right)$. We claim that then for all $\phi \in C_{0}^{\infty}\left(\left(x_{0}-\delta, x_{0}+\delta\right) ; \mathbb{R}^{2}\right)$ and $\varepsilon \in \mathbb{R}$ one has $\bar{\gamma}+\varepsilon \phi \in \mathcal{A}$. This is true since $\phi\left(x^{\prime}\right)=\phi\left(x^{\prime \prime}\right)=0$ and thus

$$
(\bar{\gamma}+\varepsilon \phi)\left(x^{\prime}\right)=\bar{\gamma}\left(x^{\prime}\right)=p_{0}=\bar{\gamma}\left(x^{\prime \prime}\right)=(\bar{\gamma}+\varepsilon \phi)\left(x^{\prime \prime}\right) .
$$


As a result we may conclude

$$
\mathcal{L}(\bar{\gamma}) D \mathcal{E}(\bar{\gamma})(\phi)+\mathcal{E}(\bar{\gamma}) D \mathcal{L}(\bar{\gamma})(\phi)=0 \quad \forall \phi \in C_{0}^{\infty}\left(\left(x_{0}-\delta, x_{0}+\delta\right) ; \mathbb{R}^{2}\right) .
$$

We have shown that for all $x_{0} \in \gamma^{-1}\left(\left\{p_{0}\right\}\right)$ there exists a neighborhood $U_{x_{0}}$ such that $\bar{\gamma}$ solves the Euler Lagrange equation weakly on $U_{x_{0}}$. By Lemma 5.8, we have $S[\bar{\gamma}]=\left\{p_{0}\right\}$ and hence we infer that for all $p_{0}=\gamma\left(x_{0}\right) \in S[\bar{\gamma}]$ there exists a neighborhood $U_{x_{0}}$ such that $\bar{\gamma}$ solves the Euler Lagrange equation weakly on $U_{x_{0}}$. Together with Lemma 5.7 we conclude that each $x \in \mathbb{S}^{1}$ has an open neighborhood $U_{x}$ such that $\bar{\gamma}$ solves (8) weakly on $U_{x}$. One can repeat the partition of unity argument in the proof of Lemma 5.8 to find that $\bar{\gamma}$ solves (8) globally in $\mathbb{S}^{1}$. Hence $\bar{\gamma}$ is by the discussion after (8) a constrained elastica. By minimality of $\bar{\gamma}$, it follows that $\bar{\gamma}$ must minimize $\mathcal{E} \mathcal{L}$ also among nonembedded elasticae and hence is a rescaled, rotated and translated reparametrization of the one-fold covered figure eight $\gamma^{*}$, cf. Lemma 5.4. As mult $\left[\gamma^{*}\right](p) \leq 2$ for all $p \in S\left[\gamma^{*}\right]$ we infer that mult $[\bar{\gamma}]\left(p_{0}\right) \leq 2$, a contradiction.

Next we show that $\bar{\gamma}$ has no tangential self-intersections. As a preparation for this, we discuss an important quantity, the winding number $T$, which is defined in Definition A.3 in Appendix A.

Proposition 5.10. Let $\gamma \in \mathcal{A}$ such that $T[\gamma] \neq \pm 1$. Then $\gamma$ is an interior point of $\mathcal{A}$ with respect to the $W^{2,2}\left(\mathbb{S}^{1} ; \mathbb{R}^{2}\right)$-norm.

Proof. Let $\gamma \in \mathcal{A}$. Since $T: W_{I m m}^{2,2}\left(\mathbb{S}^{1} ; \mathbb{R}^{2}\right) \rightarrow \mathbb{R}$ is continuous and $\mathbb{Z}$-valued, it is locally constant near $\gamma$. Thus, if $T[\gamma] \neq \pm 1$, we have $T[\tilde{\gamma}]=T[\gamma] \neq \pm 1$ for all $\tilde{\gamma} \in W^{2,2}\left(\mathbb{S}^{1} ; \mathbb{R}^{2}\right)$ with $\|\tilde{\gamma}-\gamma\|_{W^{2,2}}<\delta$ for $\delta>0$ small enough. Consequently, by Hopf's Umlaufsatz, Proposition A.5, any such $\tilde{\gamma}$ is not an embedding, hence $\tilde{\gamma} \in \mathcal{A}$.

The winding number can now be used to detect interior points of $\mathcal{A}$, which we will use next to exclude tangential self-intersections.

Lemma 5.11. Let $\bar{\gamma} \in \mathcal{A}$ be a minimizer in (5). Then $S_{\text {tan }}[\bar{\gamma}]=\emptyset$.

Proof. By Lemma 5.9, we have $S[\bar{\gamma}]=\left\{p_{0}\right\}$ with mult $[\bar{\gamma}]\left(p_{0}\right)=2$. Assume that $p_{0}$ is a tangential self-intersection with multiplicity two and $\bar{\gamma}^{-1}\left(\left\{p_{0}\right\}\right)=\left\{x_{0}, x_{1}\right\}$. After reparametrization, we may assume that $\bar{\gamma}$ is parametrized with constant speed, i.e. $\left|\bar{\gamma}^{\prime}(x)\right|=\mathcal{L}(\bar{\gamma})$ for all $x \in \mathbb{S}^{1}$. Hence, we have $\bar{\gamma}^{\prime}\left(x_{0}\right)= \pm \bar{\gamma}^{\prime}\left(x_{1}\right)$.

We first consider the case $\bar{\gamma}^{\prime}\left(x_{0}\right)=\bar{\gamma}^{\prime}\left(x_{1}\right)$. Then $\bar{\gamma}_{1}:=\left.\bar{\gamma}\right|_{\left[x_{0}, x_{1}\right]}$ and $\bar{\gamma}_{2}:=\left.\bar{\gamma}\right|_{\left[x_{1}, x_{0}\right]}$ are $C^{1}$-closed embedded curves and after an appropriate reparametrization, we have $\bar{\gamma}_{1}, \bar{\gamma}_{2} \in W^{2,2}\left(\mathbb{S}^{1} ; \mathbb{R}^{2}\right)$ using Remark 2.3 . We obtain

$$
T[\bar{\gamma}]=T\left[\bar{\gamma}_{1}\right]+T\left[\bar{\gamma}_{2}\right]
$$

Since $\bar{\gamma}_{k}$ is simple closed, we obtain $T\left[\bar{\gamma}_{k}\right]= \pm 1$ for $k=1,2$. Hence $T[\bar{\gamma}] \neq \pm 1$, so $\bar{\gamma}$ is an interior point of $\mathcal{A}$ by Proposition 5.10. Consequently $\bar{\gamma}$ satisfies (9) and thus (8). Consequently, $\bar{\gamma}$ is an elastica by the discussion after (8). Therefore, by minimality, 
must be a rescaled translated and rotated reparametrization of $\gamma^{*}$ by Lemma 5.4. This contradicts Lemma 5.6 .

For the case $\bar{\gamma}^{\prime}\left(x_{0}\right)=-\bar{\gamma}^{\prime}\left(x_{1}\right)$ we can without loss of generality assume that $x_{0}=0$ and $\bar{\gamma}^{\prime}(0)=(\mathcal{L}(\bar{\gamma}), 0)^{T}$. Let $\theta \in W^{1,2}((0,1) ; \mathbb{R})$ be the angle function from Lemma A.2 with $\theta(0)=0$. Then $\mathcal{L}(\bar{\gamma}) \kappa(x)=\theta^{\prime}(x)$ by (31). Suppose now that $\bar{\gamma}$ is not an interior point of $\mathcal{A}$. Hence $T[\bar{\gamma}]= \pm 1$ by Proposition 5.10 . After appropriate reparametrization we may assume that $T[\bar{\gamma}]=1$ and hence

$$
1=\frac{1}{2 \pi} \int_{\gamma} \kappa \mathrm{d} s=\frac{\theta(1)-\theta(0)}{2 \pi} .
$$

In particular $\theta(1)=2 \pi$. As $\theta(0)=0$ and $\bar{\gamma}^{\prime}\left(x_{1}\right)=-\bar{\gamma}^{\prime}(0)=(-\mathcal{L}(\bar{\gamma}), 0)^{T}$ we infer that $\theta\left(x_{1}\right)=k \pi$ for some odd number $k \in 2 \mathbb{Z}+1$. Now we define

$$
\widetilde{\gamma}(x):= \begin{cases}\bar{\gamma}(x) & x \in\left[0, x_{1}\right] \\ \bar{\gamma}\left(1-\left(x-x_{1}\right)\right) & x \in\left[x_{1}, 1\right] .\end{cases}
$$

The curve $\widetilde{\gamma}$ is well-defined since $\bar{\gamma}(0)=\bar{\gamma}\left(x_{1}\right)=\bar{\gamma}(1)$ using Remark 2.3. Note in particular that $x_{1} \neq 0$ in $\mathbb{S}^{1}$ and hence $\widetilde{\gamma}$ is not injective. We claim that $\widetilde{\gamma}$ is another minimizer. To this end, we show that $\widetilde{\gamma} \in W^{2,2}\left(\mathbb{S}^{1} ; \mathbb{R}^{2}\right)$ and $\mathcal{E}(\widetilde{\gamma}) \mathcal{L}(\widetilde{\gamma})=\mathcal{E}(\bar{\gamma}) \mathcal{L}(\bar{\gamma})$. By Remark 2.3 it suffices to show that the zeroth and first derivatives of the two cases coincide at $x=0=1$ and at $x=x_{1}$. This is easy to check using that $\bar{\gamma}^{\prime}(0)=\bar{\gamma}^{\prime}(1)=$ $-\bar{\gamma}^{\prime}\left(x_{1}\right)$. It is also immediate to check that $\mathcal{E}(\widetilde{\gamma})=\mathcal{E}(\bar{\gamma})$ and $\mathcal{L}(\widetilde{\gamma})=\mathcal{L}(\bar{\gamma})$. Hence $\widetilde{\gamma}$ is another minimizer as claimed. Observe also that $S_{\tan }[\widetilde{\gamma}]=S_{\tan }[\bar{\gamma}] \neq \emptyset$ since $\widetilde{\gamma}(0)=\widetilde{\gamma}\left(x_{1}\right)$ and $\widetilde{\gamma}^{\prime}(0)=-\widetilde{\gamma}^{\prime}\left(x_{1}\right)$. We now claim that

$$
\widetilde{\gamma}^{\prime}(x)=\mathcal{L}(\widetilde{\gamma})\left(\begin{array}{c}
\cos (\widetilde{\theta}(x)) \\
\sin (\widetilde{\theta}(x))
\end{array}\right) \quad \forall x \in(0,1),
$$

where $\tilde{\theta}$ is given by

$$
\tilde{\theta}(x)= \begin{cases}\theta(x) & x \in\left(0, x_{1}\right] \\ (k-2) \pi+\theta\left(1-\left(x-x_{1}\right)\right) & x \in\left(x_{1}, 1\right),\end{cases}
$$

with $k \in 2 \mathbb{Z}+1$ as before. To show that $\widetilde{\theta}$ is the angle function of $\widetilde{\gamma}$, we observe that for $x \in\left(x_{1}, 1\right)$

$$
\begin{aligned}
\widetilde{\gamma}^{\prime}(x)=-\bar{\gamma}^{\prime}\left(1-\left(x-x_{1}\right)\right) & =-\mathcal{L}(\bar{\gamma})\left(\begin{array}{c}
\cos \left(\theta\left(1-\left(x-x_{1}\right)\right)\right) \\
\sin \left(\theta\left(1-\left(x-x_{1}\right)\right)\right)
\end{array}\right) \\
& =\mathcal{L}(\bar{\gamma})\left(\begin{array}{c}
\cos \left(\pi+\theta\left(1-\left(x-x_{1}\right)\right)\right) \\
\sin \left(\pi+\theta\left(1-\left(x-x_{1}\right)\right)\right)
\end{array}\right) .
\end{aligned}
$$

Hence, by Lemma A.2, $\widetilde{\theta}$ and $\pi+\theta\left(1-\left(\cdot-x_{1}\right)\right)$ differ only by a constant multiple of $2 \pi$. The multiple has to be chosen in such a way that $\widetilde{\theta} \in W^{1,2}(0,1)$. Since $\theta\left(x_{1}\right)=k \pi=$ 
$(k-2) \pi+\theta(1)$, the only possible choice for $\widetilde{\theta}$ is the one we defined in $(19)$. Using this and the relation $\kappa\left|\gamma^{\prime}\right|=\theta^{\prime}$ (cf. (31)) we compute

$$
\begin{aligned}
\frac{1}{2 \pi} \int_{\mathbb{S}^{1}} \kappa[\tilde{\gamma}] \mathrm{d} s \tilde{\gamma} & =\frac{1}{2 \pi}\left(\int_{0}^{x_{1}} \theta^{\prime}(x) \mathrm{d} x-\int_{x_{1}}^{1} \theta^{\prime}\left(1-\left(x-x_{1}\right)\right) \mathrm{d} x\right) \\
& =\frac{1}{2 \pi}\left(\int_{0}^{x_{1}} \theta^{\prime}(y) \mathrm{d} y-\int_{x_{1}}^{1} \theta^{\prime}(y) \mathrm{d} y\right) \\
& =\frac{1}{2 \pi}\left(2 \theta\left(x_{1}\right)-\theta(0)-\theta(1)\right)=k-1,
\end{aligned}
$$

since $\theta(0)=0, \theta(1)=2 \pi$. In particular by Proposition A.4

$$
T[\widetilde{\gamma}] \notin\{+1,-1\}
$$

since $k-1$ is always even as $k$ is odd. We infer that $\widetilde{\gamma}$ is an interior point of $\mathcal{A}$. Since it is also a minimizer, it must by the same arguments as in the beginning of the proof be a rescaled, translated and reparametrized version of $\gamma^{*}$. However, this is a contradiction, since $S_{\text {tan }}\left[\gamma^{*}\right]=\emptyset$ by Lemma 5.6 but $S_{\text {tan }}[\widetilde{\gamma}]=S_{\text {tan }}[\bar{\gamma}] \neq \emptyset$.

Once we can rule out tangential self-intersections (as in Figure 2), we can finally show that a minimizer is an interior point. The following lemma shows that non-tangential self-intersections are stable under $C^{1}$-small perturbations.

Lemma 5.12. Let $\bar{\gamma} \in C^{1}\left(\mathbb{S}^{1} ; \mathbb{R}^{2}\right)$ and assume $\bar{\gamma}$ has a single non-tangential self intersection with multiplicity two, i.e. $S[\bar{\gamma}]=\{p\}$ with $\bar{\gamma}^{-1}(\{p\})=\left\{\bar{x}_{1}, \bar{x}_{2}\right\}$ for $\bar{x}_{1} \neq \bar{x}_{2}$ and $S_{\text {tan }}[\bar{\gamma}]=\emptyset$. Then, there exists $\delta>0, \varepsilon_{0}>0$ such that $\left(\bar{x}_{1}-\delta, \bar{x}_{1}+\delta\right) \cap\left(\bar{x}_{2}-\delta, \bar{x}_{2}+\delta\right)=\emptyset$ and every curve $\gamma \in B:=\left\{\eta \in C^{1}\left(\mathbb{S}^{1} ; \mathbb{R}^{2}\right) \mid\|\gamma-\bar{\gamma}\|_{C^{1}}<\varepsilon_{0}\right\}$ has a unique self intersection, i.e. there exist unique $x_{1} \neq x_{2} \in \mathbb{S}^{1}$ such that $\gamma\left(x_{1}\right)=\gamma\left(x_{2}\right)$. Moreover, this self-intersection is non-tangential, satisfies $x_{i} \in\left[\bar{x}_{i}-\delta, \bar{x}_{i}+\delta\right]$ for $i=1,2$ and the function $B \ni \gamma \mapsto\left(x_{1}, x_{2}\right) \in \mathbb{R}^{2}$ is of class $C^{1}$.

Proof. Let $\delta>0$ be small enough such that $\left(\bar{x}_{1}-\delta, \bar{x}_{1}+\delta\right) \cap\left(\bar{x}_{2}-\delta, \bar{x}_{2}+\delta\right)=\emptyset$. Moreover, taking $\varepsilon_{0}>0$ small enough and using Proposition 4.2 and Remark 4.4 , we may assume that any $\gamma \in B$ is immersed and injective when restricted to $\mathbb{S}^{1} \backslash\left(\bar{x}_{i}-\delta, \bar{x}_{i}+\delta\right)$ for $i=1,2$. Now, we define $\mathcal{U}:=\left(\bar{x}_{1}-\delta, \bar{x}_{1}+\delta\right) \times\left(\bar{x}_{2}-\delta, \bar{x}_{2}+\delta\right) \times B$ and the function

$$
\Phi: \mathcal{U} \rightarrow \mathbb{R}^{2}, \quad \Phi\left(x_{1}, x_{2}, \gamma\right):=\gamma\left(x_{1}\right)-\gamma\left(x_{2}\right) .
$$

Then $\Phi\left(\bar{x}_{1}, \bar{x}_{2}, \bar{\gamma}\right)=0$ by assumption. Moreover, $\Phi$ is of class $C^{1}$ since the map $\varphi:(a, b) \times$ $B \rightarrow \mathbb{R}^{2},(x, \gamma) \mapsto \gamma(x)$ is $C^{1}$ for any $a<b$ with derivative $D \varphi(x, \gamma)[z, \eta]=\eta(x)+$ $\gamma^{\prime}(x) z$ for $x \in(a, b), z \in \mathbb{R}, \gamma \in B$ and $\eta \in C^{1}\left(\mathbb{S}^{1} ; \mathbb{R}^{2}\right)$. Now, the partial derivative $D_{\left(x_{1}, x_{2}\right)} \Phi\left(\bar{x}_{1}, \bar{x}_{2}, \bar{\gamma}\right): \mathbb{R}^{2} \rightarrow \mathbb{R}^{2}$ given by

$$
D_{\left(x_{1}, x_{2}\right)} \Phi\left(\bar{x}_{1}, \bar{x}_{2}, \bar{\gamma}\right)\left[z_{1}, z_{2}\right]=\bar{\gamma}^{\prime}\left(\bar{x}_{1}\right) z_{1}-\bar{\gamma}^{\prime}\left(\bar{x}_{2}\right) z_{2} \quad \text { for } z_{1}, z_{2} \in \mathbb{R} .
$$

is invertible, since $\bar{\gamma}^{\prime}\left(\bar{x}_{1}\right)$ and $\bar{\gamma}^{\prime}\left(\bar{x}_{2}\right)$ are linearly independent. Since $\Phi$ is $C^{1}$, we may hence assume that

$$
D_{\left(x_{1}, x_{2}\right)} \Phi\left(x_{1}, x_{2}, \gamma\right) \text { is invertible for all }\left(x_{1}, x_{2}, \gamma\right) \in \mathcal{U}
$$


By the implicit function theorem [Zei96, Theorem 4.B], after possibly reducing $\varepsilon_{0}>0$ and $\delta>0$, for all $\gamma \in B$ there exists unique $x_{i}=x_{i}(\gamma) \in\left(\bar{x}_{i}-\delta, \bar{x}_{i}+\delta\right), i=1,2$, with $\Phi\left(x_{1}(\gamma), x_{2}(\gamma), \gamma\right)=0$ for all $\gamma \in B$. Moreover, the map $\gamma \mapsto\left(x_{1}(\gamma), x_{2}(\gamma)\right)$ is of class $C^{1}$. The uniqueness of the self-intersection follows from the local uniqueness and the fact that $\gamma$ is injective on $\mathbb{S}^{1} \backslash\left(\bar{x}_{i}-\delta, \bar{x}_{i}+\delta\right)$ for both $i=1$ and $i=2$. Furthermore, by (20) this self-intersection is always non-tangential.

Equipped with this result, we can show that our minimizer satisfies the Euler-Lagrange equation.

Lemma 5.13. Let $\bar{\gamma} \in \mathcal{A}$ be a minimizer in (5). Then $\bar{\gamma}$ is a constrained elastica.

Proof. By Lemma 5.9 and Lemma 5.11, there exist exactly two values $x_{1} \neq x_{2} \in \mathbb{S}^{1}$ with $\bar{\gamma}\left(x_{1}\right)=\bar{\gamma}\left(x_{2}\right)=p_{0}$ such that $\bar{\gamma}^{\prime}\left(x_{1}\right)$ and $\bar{\gamma}^{\prime}\left(x_{2}\right)$ are linearly independent. Let $\phi \in$ $C_{0}^{\infty}\left(\mathbb{S}^{1} ; \mathbb{R}^{2}\right)$. By Lemma 5.12 , there exists $\varepsilon_{0}>0$ such that for all $\varepsilon \in\left(-\varepsilon_{0}, \varepsilon_{0}\right)$ the curve $\bar{\gamma}+\varepsilon \phi$ has a self-intersection, so $\bar{\gamma}+\varepsilon \phi \in \mathcal{A}$. Then, $\left(-\varepsilon_{0}, \varepsilon_{0}\right) \ni \varepsilon \mapsto \mathcal{E}(\bar{\gamma}+\varepsilon \phi) \mathcal{L}(\bar{\gamma}+\varepsilon \phi)$ has a local minimum in $\varepsilon=0$. We conclude

$$
\mathcal{L}(\bar{\gamma}) D \mathcal{E}(\bar{\gamma})(\phi)+\mathcal{E}(\bar{\gamma}) D \mathcal{L}(\bar{\gamma})(\phi)=0 \quad \forall \phi \in C_{0}^{\infty}\left(\mathbb{S}^{1} ; \mathbb{R}^{2}\right)
$$

Finally, we can prove our main result.

Proof of Theorem 1.2. By Theorem 4.5 there exists $\bar{\gamma} \in \mathcal{A}$ such that

$$
\mathcal{E}(\bar{\gamma}) \mathcal{L}(\bar{\gamma})=\inf _{\gamma \in \mathcal{A}} \mathcal{E}(\gamma) \mathcal{L}(\gamma)
$$

By Lemma 5.13 we infer that $\bar{\gamma} \in \mathcal{B}$, where $\mathcal{B}$ is defined as in 10$)$. This implies together with Lemma 5.4 that

$$
\inf _{\gamma \in \mathcal{A}} \mathcal{E}(\gamma) \mathcal{L}(\gamma)=\mathcal{E}(\bar{\gamma}) \mathcal{L}(\bar{\gamma})=\inf _{\gamma \in \mathcal{B}} \mathcal{E}(\gamma) \mathcal{L}(\gamma)=\mathcal{E}\left(\gamma^{*}\right) \mathcal{L}\left(\gamma^{*}\right)
$$

The claim follows.

Remark 5.14. If we seek to generalize Theorem 1.2 in higher codimension, e.g. in $\mathbb{R}^{3}$, the arguments in this section do not immediately carry over as more elasticae would need to be discussed in a generalized version of Lemma 5.4.

An exhaustive classification of elasticae in $\mathbb{R}^{3}$ in [LS84b] shows that all non-planar elasticae $\gamma$ are embedded and knotted. Therefore, by the Fary-Milnor Theorem (cf. [Fár49, Mil50]) their energy is bounded from below since

$$
\mathcal{E}(\gamma) \mathcal{L}(\gamma)=\int_{\mathbb{S}^{1}}|\kappa|^{2} \mathrm{~d} s \int_{\mathbb{S}^{1}} 1 \mathrm{~d} s \geq\left(\int_{\mathbb{S}^{1}}|\kappa| \mathrm{d} s\right)^{2} \geq 16 \pi^{2}>c^{*},
$$

where we used (16) in the last inequality. Clearly, multi-fold covers of these non-planar elasticae will only have higher energy. Hence, $\gamma^{*}$ is still the minimizer among all nonembedded elasticae in $\mathbb{R}^{3}$, generalizing Lemma 5.4 to higher codimension. Moreover, we can prove as in Lemma 5.8 and Lemma 5.9 that there exists a minimizer $\bar{\gamma}$ of $\mathcal{E} \mathcal{L}$ 
among closed non-embedded curves in $\mathbb{R}^{3}$ which has only one point of self-intersection with multiplicity 2. However, the methods used in Lemma 5.11 and Lemma 5.13 are only available in codimension one, making it unclear, whether the minimizer is an elastica. This is the only obstruction to a generalization of Theorem 1.2 into higher codimension.

\section{An application: the elastic flow}

We consider a family of smooth curves $\gamma:[0, T) \times \mathbb{S}^{1} \rightarrow \mathbb{R}^{2}$ evolving with respect to the gradient flow equation

$$
\partial_{t} \gamma=-\nabla_{s}^{2} \vec{\kappa}-\frac{1}{2}|\vec{\kappa}|^{2} \vec{\kappa}+\lambda \vec{\kappa}
$$

where $\vec{\kappa}=\vec{\kappa}[\gamma]=\partial_{s}^{2} \gamma$ is the curvature vector and $\nabla_{s}=\partial_{s}^{\perp \gamma}$ is the normal part of the arc-length derivative. Here, we either consider the length penalized elastic flow, where $\lambda \geq 0$ is a fixed number or the length preserving elastic flow, where $\lambda=\lambda(\gamma(t, \cdot)) \in \mathbb{R}$ depends on the solution and is given by

$$
\lambda=\frac{\int_{\mathbb{S}^{1}}\left\langle\nabla_{s}^{2} \vec{\kappa}+\frac{1}{2}|\vec{\kappa}|^{2} \vec{\kappa}, \vec{\kappa}\right\rangle \mathrm{d} s}{\int_{\mathbb{S}^{1}}|\vec{\kappa}|^{2} \mathrm{~d} s} .
$$

It can be easily checked that the length remains constant along solutions of (21) with $\lambda$ given by $(22)$, since

$$
\frac{\mathrm{d}}{\mathrm{d} t} \mathcal{L}(\gamma)=\int\left\langle\nabla \mathcal{L}(\gamma), \partial_{t} \gamma\right\rangle \mathrm{d} s=-\int_{\mathbb{S}^{1}}\left\langle\vec{\kappa}, \partial_{t} \gamma\right\rangle \mathrm{d} s=0,
$$

by (22). Both geometric flows have been studied in [DKS02], where long-time existence and subconvergence as $t \rightarrow \infty$ has been established. Using Theorem 1.2 , we establish an energy bound which guarantees embeddedness along the flow. Note that in contrast to second order evolutions, this does not follow from a maximum principle, as (21) is of 4-th order. Moreover as in [DPS16, MP20, RS20] we can apply a suitable Eojasiewicz-Simon inequality to deduce convergence and then Lemma 5.4 to give a precise characterization of the limit.

Theorem 6.1. Let $\gamma_{0} \in C^{\infty}\left(\mathbb{S}^{1} ; \mathbb{R}^{2}\right)$ be an embedded curve such that $\mathcal{E}\left(\gamma_{0}\right) \mathcal{L}\left(\gamma_{0}\right)<$ $c^{*}$. Then the elastic flow (21) with fixed length and initial datum $\gamma(0, \cdot)=\gamma_{0}$ remains embedded for all times and converges, as $t \rightarrow \infty$, after reparametrization with constant speed to a one-fold cover of a circle with radius $\frac{\mathcal{L}\left(\gamma_{0}\right)}{2 \pi}$.

Proof. We write $\gamma(t):=\gamma(t, \cdot)$ and observe that the energy $\mathcal{E}(\gamma(t))$ is decreasing, while $\mathcal{L}(\gamma(t))=\mathcal{L}\left(\gamma_{0}\right)$ is kept fixed. Hence for $t \geq 0$, we have $\mathcal{E}(\gamma(t)) \mathcal{L}(\gamma(t))<\mathcal{E}\left(\gamma^{*}\right) \mathcal{L}\left(\gamma^{*}\right)$ by the assumption on the initial datum, so $\gamma(t)$ is embedded for all $t \geq 0$ by Theorem 1.2 . Now, by [DKS02, Theorem 3.3], the flow exists for all times and it holds $\left\|\partial_{s}^{m} \vec{\kappa}\right\|_{L^{\infty}} \leq C_{m}$, for some $C_{m}>0$ and all $m \in \mathbb{N}_{0}$. Thus, if $\tilde{\gamma}$ denotes the reparametrization by arc-length, we get

$$
\left\|\partial_{x}^{m} \tilde{\gamma}\right\|_{L^{\infty}} \leq C_{m} \text { for all } m \in \mathbb{N} \text {. }
$$


If we define the integral average $p(t):=\int_{\mathbb{S}^{1}} \tilde{\gamma}(t, \cdot) \mathrm{d} x \in \mathbb{R}^{2}$, we find

$$
\|\tilde{\gamma}(t, \cdot)-p(t)\|_{L^{\infty}} \leq \mathcal{L}(\tilde{\gamma}(t))=\mathcal{L}\left(\gamma_{0}\right) .
$$

Now, if $t_{n} \rightarrow \infty$ is any sequence, using the Arzelà-Ascoli theorem and a diagonal sequence argument, after passing to a subsequence, we find $\tilde{\gamma}\left(t_{n}\right)-p\left(t_{n}\right) \rightarrow \gamma_{\infty}$ in $C^{m}\left(\mathbb{S}^{1} ; \mathbb{R}^{2}\right)$ as $n \rightarrow \infty$ for every $m \in \mathbb{N}_{0}$, where $\gamma_{\infty} \in C^{\infty}\left(\mathbb{S}^{1} ; \mathbb{R}^{2}\right)$ is a closed elastica, cf. DKS02, Theorem 3.2]. Since $\mathcal{E}\left(\gamma_{\infty}\right) \mathcal{L}\left(\gamma_{\infty}\right)<\mathcal{E}\left(\gamma^{*}\right) \mathcal{L}\left(\gamma^{*}\right)$, the curve $\gamma_{\infty}$ is embedded and has to be a (translation and reparametrization) of the one-fold cover of a circle by Lemma 5.4. Consequently, its radius has to be $\frac{\mathcal{L}\left(\gamma_{0}\right)}{2 \pi}$ since $\mathcal{L}\left(\gamma_{\infty}\right)=\mathcal{L}\left(\gamma_{0}\right)$. However, different sequences could still yield circles with different centers.

Even so, this cannot happen, since $\mathcal{E}$ satisfies a constrained Łojasiewicz-Simon gradient inequality, cf. Rup20. This can be proven using [Rup20, Corollary 5.2], since the energies $\mathcal{E}$ and $\mathcal{L}$ are analytic and the length is of lower order, see also [RS20, Theorem 4.8] for the analogous argument in the case of clamped curves. Hence, there exist constants $C_{L S}, \sigma>0$ and $\theta \in\left(0, \frac{1}{2}\right]$ such that for all $\gamma \in W^{4,2}\left(\mathbb{S}^{1} ; \mathbb{R}^{2}\right)$ with $\left\|\gamma-\gamma_{\infty}\right\|_{W^{4,2}} \leq \sigma$ and $\mathcal{L}(\gamma)=\mathcal{L}\left(\gamma_{0}\right)$ we have

$$
\left|\mathcal{E}(\gamma)-\mathcal{E}\left(\gamma_{\infty}\right)\right|^{1-\theta} \leq C\|\nabla \mathcal{E}(\gamma)+\lambda(\gamma) \nabla \mathcal{L}(\gamma)\|_{L^{2}},
$$

with $\lambda(\gamma)$ as in $(22)$. To prove the full convergence statement, we furthermore assume $\left\|\tilde{\gamma}\left(t_{n}\right)-p\left(t_{n}\right)-\gamma_{\infty}\right\|_{W^{4,2}}<\sigma$ for all $n \in \mathbb{N}$. We define

$$
s_{n}:=\sup \left\{s \geq t_{n} \mid\left\|\tilde{\gamma}(t)-p(t)-\gamma_{\infty}\right\|_{W^{4,2}}<\sigma \text { for all } t \in\left[t_{n}, s\right]\right\},
$$

and observe $s_{n}>t_{n}$ by smoothness. Now, the function

$$
G(t):=\left(\mathcal{E}(\tilde{\gamma}(t))-\mathcal{E}\left(\gamma_{\infty}\right)\right)^{\theta}=\left(\mathcal{E}(\gamma(t))-\mathcal{E}\left(\gamma_{\infty}\right)\right)^{\theta}
$$

is decreasing and satisfies $\lim _{t \rightarrow \infty} G(t)=0$. Moreover, since $\gamma$ solves 21) we have

$$
\begin{aligned}
-\frac{\mathrm{d}}{\mathrm{d} t} G & =\theta\left(\mathcal{E}(\tilde{\gamma})-\mathcal{E}\left(\gamma_{\infty}\right)\right)^{\theta-1}\left(-\frac{\mathrm{d}}{\mathrm{d} t} \mathcal{E}(\gamma)\right) \\
& =-\theta\left(\mathcal{E}(\tilde{\gamma})-\mathcal{E}\left(\gamma_{\infty}\right)\right)^{\theta-1}\left\langle\nabla \mathcal{E}(\gamma), \partial_{t} \gamma\right\rangle_{L^{2}\left(\mathrm{~d} s_{\gamma}\right)} \\
& =-\theta\left(\mathcal{E}(\tilde{\gamma})-\mathcal{E}\left(\gamma_{\infty}\right)\right)^{\theta-1}\left\langle\nabla \mathcal{E}(\gamma)+\lambda(\gamma) \nabla \mathcal{L}(\gamma), \partial_{t} \gamma\right\rangle_{L^{2}\left(\mathrm{~d} s_{\gamma}\right)} \\
& =\theta\left(\mathcal{E}(\tilde{\gamma})-\mathcal{E}\left(\gamma_{\infty}\right)\right)^{\theta-1}\|\nabla \mathcal{E}(\gamma)+\lambda(\gamma) \nabla \mathcal{L}(\gamma)\|_{L^{2}\left(\mathrm{~d} s_{\gamma}\right)}\left\|\partial_{t} \gamma\right\|_{L^{2}\left(\mathrm{~d} s_{\gamma}\right)}
\end{aligned}
$$

where we used that $\left\langle\nabla \mathcal{L}(\gamma), \partial_{t} \gamma\right\rangle_{L^{2}\left(\mathrm{~d} s_{\gamma}\right)}=0$ by 23. Furthermore, using the geometric transformation of the energy and the $L^{2}$-gradient, we find

$$
\begin{aligned}
& \left(\mathcal{E}(\tilde{\gamma})-\mathcal{E}\left(\gamma_{\infty}\right)\right)^{\theta-1}\|\nabla \mathcal{E}(\gamma)+\lambda(\gamma) \nabla \mathcal{L}(\gamma)\|_{L^{2}\left(\mathrm{~d} s_{\gamma}\right)} \\
& \quad=\left(\mathcal{E}(\tilde{\gamma}-p)-\mathcal{E}\left(\gamma_{\infty}\right)\right)^{\theta-1}\|\nabla \mathcal{E}(\tilde{\gamma}-p)+\lambda(\tilde{\gamma}-p) \nabla \mathcal{L}(\tilde{\gamma}-p)\|_{L^{2}\left(\mathrm{~d} s_{\tilde{\gamma}-p}\right)} .
\end{aligned}
$$

Thus, by the definition of $s_{n}$ and the Łojasiewicz-Simon inequality (26) we have

$$
-\frac{\mathrm{d}}{\mathrm{d} t} G(t) \geq \frac{\theta}{C_{L S}}\left\|\partial_{t} \gamma(t)\right\|_{L^{2}\left(\mathrm{~d} s_{\gamma(t)}\right)} \quad \text { for all } t \in\left[t_{n}, s_{n}\right) .
$$


Consequently, by [RS20, Lemma 4.10 and Remark 4.11], we find

$$
-\frac{\mathrm{d}}{\mathrm{d} t} G(t) \geq C\left\|\partial_{t} \tilde{\gamma}(t)\right\|_{L^{2}(\mathrm{~d} x)} \quad \text { for all } t \in\left[t_{n}, s_{n}\right)
$$

for some $C=C\left(\mathcal{L}\left(\gamma_{0}\right), \mathcal{E}\left(f_{0}\right), \theta, C_{L S}\right)>0$. Thus, given $\varepsilon>0$ and $t \in\left[t_{n}, s_{n}\right)$ we obtain

$$
\left\|\tilde{\gamma}(t)-\tilde{\gamma}\left(t_{n}\right)\right\|_{L^{2}(\mathrm{~d} x)} \leq \frac{1}{C} G\left(t_{n}\right) \rightarrow 0,
$$

as $n \rightarrow \infty$ since $\lim _{n \rightarrow \infty} G\left(t_{n}\right)=0$. Now, we assume that all of the $s_{n}$ are finite. By continuity, (28) also holds for $t=s_{n}$ and we observe

$$
\begin{aligned}
\left\|p\left(t_{n}\right)-p\left(s_{n}\right)\right\|_{L^{2}(\mathrm{~d} x)}^{2} & =\left|\int_{\mathbb{S}^{1}} \tilde{\gamma}\left(t_{n}\right) \mathrm{d} x-\int_{\mathbb{S}^{1}} \tilde{\gamma}\left(s_{n}\right) \mathrm{d} x\right|^{2} \\
& \leq \int_{\mathbb{S}^{1}}\left|\tilde{\gamma}\left(t_{n}\right)-\tilde{\gamma}\left(s_{n}\right)\right|^{2} \mathrm{~d} x \rightarrow 0,
\end{aligned}
$$

using Jensen's inequality and (28). Moreover, using the bounds in (24) and (25) we may, as at the beginning of the proof, assume that $\tilde{\gamma}\left(s_{n}\right)-p\left(s_{n}\right) \rightarrow \psi$ smoothly as $n \rightarrow \infty$ for some $\psi \in C^{\infty}\left(\mathbb{S}^{1} ; \mathbb{R}^{2}\right)$. Thus, we find

$$
\begin{aligned}
\left\|\tilde{\gamma}\left(s_{n}\right)-p\left(s_{n}\right)-\gamma_{\infty}\right\|_{L^{2}(\mathrm{~d} x) \leq} \leq & \left\|\tilde{\gamma}\left(s_{n}\right)-\tilde{\gamma}\left(t_{n}\right)\right\|_{L^{2}(\mathrm{~d} x)}+\left\|p\left(t_{n}\right)-p\left(s_{n}\right)\right\|_{L^{2}(\mathrm{~d} x)} \\
& +\left\|\tilde{\gamma}\left(t_{n}\right)-p\left(t_{n}\right)-\gamma_{\infty}\right\|_{L^{2}(\mathrm{~d} x)} \rightarrow 0, \text { as } n \rightarrow \infty .
\end{aligned}
$$

Therefore, $\psi=f_{\infty}$. However, by the definition of $s_{n}$ and a continuity argument, we have

$$
\left\|\psi-f_{\infty}\right\|_{W^{4,2}}=\lim _{n \rightarrow \infty}\left\|\tilde{\gamma}\left(s_{n}\right)-p\left(s_{n}\right)-\gamma_{\infty}\right\|_{W^{4,2}}=\sigma>0
$$

a contradiction. Consequently, we have $s_{n_{0}}=\infty$ for some $n_{0} \in \mathbb{N}$ and therefore $\left\|\tilde{\gamma}(t)-p(t)-\gamma_{\infty}\right\|_{W^{4,2}}<\sigma$ for all $t \geq t_{n_{0}}$. But then 27) implies that for any $t_{n_{0}} \leq t \leq t^{\prime}$ we have

$$
\left\|\tilde{\gamma}(t)-\tilde{\gamma}\left(t^{\prime}\right)\right\|_{L^{2}(\mathrm{~d} x)} \leq \int_{t}^{t^{\prime}}\left\|\partial_{t} \tilde{\gamma}(\tau)\right\|_{L^{2}(\mathrm{~d} x)} \mathrm{d} \tau \rightarrow 0
$$

as $t^{\prime}, t \rightarrow \infty$ by dominated convergence. Therefore, the $\operatorname{limit}_{t \rightarrow \infty} \tilde{\gamma}(t)$ has to exist in $L^{2}(\mathrm{~d} x)$ and hence equals $\gamma_{\infty}$. A subsequence argument shows that for any $m \in \mathbb{N}_{0}$ we have $\left\|\tilde{\gamma}(t)-\gamma_{\infty}\right\|_{\mathcal{C}^{m}\left(I ; \mathbb{R}^{d}\right)} \rightarrow 0$ as $t \rightarrow \infty$, i.e. the convergence is smooth.

The bound in Theorem 6.1 is optimal, since the stationary flow $\gamma(t) \equiv \gamma^{*}$ with $\gamma^{*}$ as in Definition 5.1 solves (21) but possesses self-intersections for all times.

Theorem 6.2. Let $\lambda>0$ and let $\gamma_{0} \in C^{\infty}\left(\mathbb{S}^{1} ; \mathbb{R}^{2}\right)$ be an embedded curve such that $\frac{\left(\mathcal{E}\left(\gamma_{0}\right)+\lambda \mathcal{L}\left(\gamma_{0}\right)\right)^{2}}{4 \lambda}<c^{*}$. Then, the length penalized elastic flow 21) remains embedded for all times and converges, as $t \rightarrow \infty$, after reparametrization to a one-fold cover of a circle with radius $\frac{1}{\sqrt{2 \lambda}}$. 
Proof. By [DKS02, Theorem 3.2] the flow exists for all times and also satisfies the bounds (24) and (25). Moreover, we have the bounds

$$
\mathcal{E}(\gamma(t))+\lambda \mathcal{L}(\gamma(t)) \leq \mathcal{E}\left(\gamma_{0}\right)+\lambda \mathcal{L}\left(\gamma_{0}\right)
$$

for all $t \geq 0$. An easy calculation yields $\max \{x y \mid x, y \in(0, \infty), x+\lambda y \leq M\}=\frac{M^{2}}{4 \lambda}$ for any $M>0$, so we have $\mathcal{E}(\gamma(t)) \mathcal{L}(\gamma(t)) \leq \frac{\left(\mathcal{E}\left(\gamma_{0}\right)+\lambda \mathcal{L}\left(\gamma_{0}\right)^{2}\right)}{4 \lambda}<c^{*}$ by assumption. Thus, using Theorem 1.2 the flow remains embedded.

As in the proof of Theorem 6.1, by the uniform estimates (24) and (25), there exists $t_{n} \rightarrow \infty$ such that $\tilde{\gamma}\left(t_{n}\right)-p\left(t_{n}\right) \rightarrow \gamma_{\infty}$, where $\tilde{\gamma}$ is a reparametrization of $\gamma$ and $\gamma_{\infty}$ is a closed elastica. Here $p(t)=\int_{\mathbb{S}^{1}} \tilde{\gamma}(t) \mathrm{d} x \in \mathbb{R}^{2}$. The convergence of the flow as $t \rightarrow \infty$ has been established in [MP20, Theorem 1.2 and Remark 1.4]. Alternatively, one can easily modify the arguments in the proof of Theorem 6.1 and apply a (unconstrained) Łojasiewicz-Simon gradient inequality for the penalized elastic energy.

Since the limit is necessarily an elastica with $\mathcal{E}\left(\gamma_{\infty}\right) \mathcal{L}\left(\gamma_{\infty}\right)<c^{*}$, it can only be a one-fold cover of a circle by Lemma 5.4. Denoting by $R>0$ its radius, we observe

$$
\partial_{s}^{2} \kappa+\frac{1}{2} \kappa^{3}-\lambda \kappa=\frac{1}{2 R^{3}}-\frac{\lambda}{R}
$$

which equals zero if and only if $R=\frac{1}{\sqrt{2 \lambda}}$.

\section{Acknowledgments}

Marius Müller was supported by the LGFG Grant (Grant no. 1705 LGFG-E). Fabian Rupp is supported by the Deutsche Forschungsgemeinschaft (DFG, German Research Foundation) - project no. 404870139. Both authors would like to thank Anna Dall'Acqua for helpful discussions.

\section{Appendix A Differential geometry in Sobolev spaces}

In this section, we will review some standard results from elementary differential geometry in the setting of $W^{2,2}$-curves.

Theorem A.1 (Fenchel's Theorem). Let $\gamma \in C^{2}\left(\mathbb{S}^{1} ; \mathbb{R}^{2}\right)$ be an immersed curve. Then $\mathcal{K}(\gamma) \geq 2 \pi$ with equality if and only if $\gamma$ is embedded and convex.

Proof. See [Fen29, Satz 1]. An explicit characterization of the equality case can be deduced from [BH74, Theorem 3], for instance.

Lemma A.2 (Angle Function). For an immersed curve $\gamma \in W^{2,2}\left(\mathbb{S}^{1} ; \mathbb{R}^{2}\right)$, there exists $\theta \in W^{1,2}((0,1) ; \mathbb{R})$ such that

$$
\frac{\gamma^{\prime}(x)}{\left|\gamma^{\prime}(x)\right|}=\left(\begin{array}{c}
\cos \theta(x) \\
\sin \theta(x)
\end{array}\right) \text { for all } x \in \mathbb{S}^{1} .
$$

We call $\theta$ an angle function for $\gamma$. Moreover, any two functions satisfying (29) can only differ by an integer multiple of $2 \pi$. 
Proof. The proof works exactly as in the case of smooth curves, see for instance Bär10, Lemma 2.2.5]. For the regularity of $\theta$, we use local representations of $\theta$. For instance, in the case $\gamma_{1}^{\prime}(x)>0$, one has locally

$$
\theta(x):=\arctan \left(\frac{\gamma_{2}^{\prime}(x)}{\gamma_{1}^{\prime}(x)}\right)+2 \pi \ell, \quad \text { for some } \ell \in \mathbb{Z} .
$$

Hence $\theta \in W^{1,2}\left(\mathbb{S}^{1} ; \mathbb{R}^{2}\right)$ follows from $(30)$ and the chain rule for Sobolev functions.

Definition A.3 (Winding Number). Let $\gamma \in W^{2,2}\left(\mathbb{S}^{1} ; \mathbb{R}^{2}\right)$ be an immersion with corresponding angle function $\theta \in W^{1,2}((0,1) ; \mathbb{R})$. We define the winding number of $\gamma$ as $T[\gamma]:=\frac{1}{2 \pi}(\theta(1)-\theta(0))$. Note that $T[\gamma]$ does not depend on the choice of $\theta$ and is always an integer.

Proposition A.4. Let $\gamma \in W^{2,2}\left(\mathbb{S}^{1} ; \mathbb{R}^{2}\right)$ be an immersed curve. Then

$$
T[\gamma]=\frac{1}{2 \pi} \int_{\mathbb{S}^{1}} \kappa \mathrm{d} s
$$

Proof. Let $\theta \in W^{1,2}((0,1) ; \mathbb{R})$ be an angle function for $\gamma$. Differentiating 29 and using the chain rule for Sobolev functions and the definition of the unit normal, we obtain

$$
\kappa \vec{n}=\vec{\kappa}=\partial_{s}^{2} \gamma=\frac{\theta^{\prime}}{\left|\gamma^{\prime}\right|}\left(\begin{array}{c}
-\sin \theta \\
\cos \theta
\end{array}\right)=\frac{\theta^{\prime}}{\left|\gamma^{\prime}\right|}\left(\begin{array}{c}
-\gamma_{2}^{\prime} \\
\gamma_{1}^{\prime}
\end{array}\right)=\frac{\theta^{\prime}}{\left|\gamma^{\prime}\right|} \vec{n},
$$

consequently

$$
\kappa\left|\gamma^{\prime}\right|=\theta^{\prime} \text { almost everywhere. }
$$

Moreover, by the fundamental theorem of calculus for $W^{1,2}$-functions, we find

$$
T[\gamma]=\frac{1}{2 \pi}(\theta(1)-\theta(0))=\frac{1}{2 \pi} \int_{0}^{1} \theta^{\prime} \mathrm{d} x=\frac{1}{2 \pi} \int_{0}^{1} \kappa\left|\gamma^{\prime}\right| \mathrm{d} x=\frac{1}{2 \pi} \int_{\mathbb{S}^{1}} \kappa \mathrm{d} s .
$$

Proposition A.5 (Hopf's Umlaufsatz for $W^{2,2}$-embeddings). Let $\gamma \in W^{2,2}\left(\mathbb{S}^{1} ; \mathbb{R}^{2}\right)$ be an embedding. Then $T[\gamma]= \pm 1$.

Proof. Let $\gamma^{(n)}$ be a sequence of smooth curves with $\gamma^{(n)} \rightarrow \gamma$ in $W^{2,2}\left(\mathbb{S}^{1} ; \mathbb{R}^{2}\right)$. By Proposition A.4, we can easily see that $T\left[\gamma^{(n)}\right] \rightarrow T[\gamma]$. Since the set of embeddings is open in $C^{1}\left(\mathbb{S}^{1} ; \mathbb{R}^{2}\right)$ by Lemma 4.3 , we see that $\gamma^{(n)}$ is an embedding for $n \geq N$ large enough, hence $T\left[\gamma^{(n)}\right]= \pm 1$ for all $n \geq N$ by Hopf's Umlaufsatz for smooth curves. However, since the sequence $\left(T\left[\gamma^{(n)}\right]\right)_{n \in \mathbb{N}}$ converges, it has to be eventually constant, say $T\left[\gamma^{(n)}\right]=\tau \in\{-1,1\}$ for all $n \geq N$. But then $T[\gamma]=\lim _{n \rightarrow \infty} T\left[\gamma^{(n)}\right]=\tau \in\{-1,1\}$.

Lemma A.6. Let $\gamma \in W^{2,2}\left(\mathbb{S}^{1} ; \mathbb{R}^{2}\right)$ be an immersion. Then, there exists a constant speed reparametrization $\tilde{\gamma}$ of $\gamma$ such that $\tilde{\gamma} \in W^{2,2}\left(\mathbb{S}^{1} ; \mathbb{R}^{2}\right)$.

Proof. Follows with the arguments in [Bär10, Proposition 2.1.13], using the Sobolev embedding $W^{2,2}\left(\mathbb{S}^{1} ; \mathbb{R}^{2}\right) \hookrightarrow C^{1}\left(\mathbb{S}^{1} ; \mathbb{R}^{2}\right)$ and the chain rule for Sobolev functions. 


\section{Appendix B Jacobi elliptic functions and Euler's elastica}

\section{B.1 Elliptic functions}

We provide some elementary properties of Jacobi elliptic functions, which can be found for example in [AS64, Chapter 16].

Definition B.1 (Amplitude Function, Complete Elliptic Integrals). Fix $m \in[0,1)$. We define the Jacobi-amplitude function $\operatorname{am}(\cdot, m): \mathbb{R} \rightarrow \mathbb{R}$ with modulus $m$ to be the inverse function of

$$
\mathbb{R} \ni z \mapsto \int_{0}^{z} \frac{1}{\sqrt{1-m \sin ^{2}(\theta)}} \mathrm{d} \theta \in \mathbb{R}
$$

We define the complete elliptic integral of first and second kind as

$$
K(m):=\int_{0}^{\frac{\pi}{2}} \frac{1}{\sqrt{1-m \sin ^{2}(\theta)}} \mathrm{d} \theta, \quad E(m):=\int_{0}^{\frac{\pi}{2}} \sqrt{1-m \sin ^{2}(\theta)} \mathrm{d} \theta
$$

and the incomplete elliptic integral of first and second kind as

$$
F(x, m):=\int_{0}^{x} \frac{1}{\sqrt{1-m \sin ^{2}(\theta)}} \mathrm{d} \theta, \quad E(x, m):=\int_{0}^{x} \sqrt{1-m \sin ^{2}(\theta)} \mathrm{d} \theta .
$$

Note that $F(\cdot, m)=\operatorname{am}(\cdot, m)^{-1}$.

Definition B.2 (Elliptic Functions). For $m \in[0,1)$ the Jacobi elliptic functions are given by

$$
\begin{aligned}
& \operatorname{cn}(\cdot, m): \mathbb{R} \rightarrow \mathbb{R}, \operatorname{cn}(x, m):=\cos (\operatorname{am}(x, m)), \\
& \operatorname{sn}(\cdot, m): \mathbb{R} \rightarrow \mathbb{R}, \operatorname{sn}(x, m):=\sin (\operatorname{am}(x, m)), \\
& \operatorname{dn}(\cdot, m): \mathbb{R} \rightarrow \mathbb{R}, \operatorname{dn}(x, m):=\sqrt{1-m \sin ^{2}(\operatorname{am}(x, m))} .
\end{aligned}
$$

The following proposition summarizes all relevant properties and identities for the elliptic functions. They can all be found in [AS64, Chapter 16].

\section{Proposition B.3.}

1. (Derivatives and Integrals of Jacobi Elliptic Functions) For each $x \in \mathbb{R}$ and $m \in$ $(0,1)$ we have

$$
\begin{aligned}
\frac{\partial}{\partial x} \operatorname{cn}(x, m) & =-\operatorname{sn}(x, m) \operatorname{dn}(x, m), & \frac{\partial}{\partial x} \operatorname{sn}(x, m) & =\operatorname{cn}(x, m) \operatorname{dn}(x, m), \\
\frac{\partial}{\partial x} \operatorname{dn}(x, m) & =-m \operatorname{cn}(x, m) \operatorname{sn}(x, m), & \frac{\partial}{\partial x} \operatorname{am}(x, m) & =\operatorname{dn}(x, m) .
\end{aligned}
$$

2. (Derivatives of Complete Elliptic Integrals) For $m \in(0,1) E$ is smooth and

$$
\frac{\mathrm{d}}{\mathrm{d} m} E(m)=\frac{E(m)-K(m)}{2 m}, \quad \frac{\mathrm{d}}{\mathrm{d} m} K(m)=\frac{(m-1) K(m)+E(m)}{2 m(1-m)} .
$$


3. (Trigonometric Identities) For each $m \in[0,1)$ and $x \in \mathbb{R}$ the Jacobi elliptic functions satisfy

$$
\operatorname{cn}^{2}(x, m)+\operatorname{sn}^{2}(x, m)=1, \quad \operatorname{dn}^{2}(x, m)+m \operatorname{sn}^{2}(x, m)=1 .
$$

4. (Periodicity) All periods of the elliptic functions are given as follows, where $l \in \mathbb{Z}$ and $x \in \mathbb{R}$ :

$$
\begin{aligned}
& \operatorname{am}(l K(m), m)=l \frac{\pi}{2}, \quad \operatorname{cn}(x+4 l K(m), m)=\operatorname{cn}(x, m), \\
& \operatorname{sn}(x+4 l K(m), m)=\operatorname{sn}(x, m), \quad \operatorname{dn}(x+2 l K(m), m)=\operatorname{dn}(x, m), \\
& F\left(\frac{l \pi}{2}, m\right)=l K(m) \quad E\left(\frac{l \pi}{2}, m\right)=l E(m) \\
& \operatorname{am}(x+2 l K(m), m)=l \pi+\operatorname{am}(x, m), \\
& F(x+l \pi, m)=F(x, m)+2 l K(m), \\
& E(x+l \pi, m)=E(x, m)+2 l E(m) .
\end{aligned}
$$

5. (Asymptotics of the Complete Elliptic Integrals)

$$
\begin{array}{ll}
\lim _{m \rightarrow 1} K(m)=\infty, & \lim _{m \rightarrow 0} K(m)=\frac{\pi}{2} \\
\lim _{m \rightarrow 1} E(m)=1, & \lim _{m \rightarrow 0} E(m)=\frac{\pi}{2} .
\end{array}
$$

\section{B.2 Some computational lemmas involving elliptic functions}

We will also need some more advanced identities for elliptic functions, e.g the following

Lemma B.4. The map $(0,1) \ni m \mapsto 2 E(m)-K(m)$ has a unique zero $m^{*} \in(0,1)$. Moreover $m^{*}>\frac{1}{2}$.

Proof. We define for $m \in(0,1), f(m):=\frac{2 E(m)}{K(m)}-1$. Note that $f$ has the same zeroes as $m \mapsto 2 E(m)-K(m)$. By Proposition B.3 one has

$$
\lim _{m \rightarrow 0} f(m)=1, \quad \lim _{m \rightarrow 1} f(m)=-1 .
$$

and hence there has to exists a zero of $f$. To show that it is unique, we show that $f$ is decreasing, which follows immediately from the following computation

$$
\begin{aligned}
\frac{\mathrm{d}}{\mathrm{d} m} \frac{E(m)}{K(m)} & =\frac{1}{K(m)^{2}}\left(\frac{E(m)-K(m)}{2 m} K(m)-E(m) \frac{(m-1) K(m)+E(m)}{2 m(1-m)}\right) \\
& =\frac{1}{2 m(1-m) K(m)^{2}}\left(2(1-m) E(m) K(m)-(1-m) K(m)^{2}-E(m)^{2}\right)
\end{aligned}
$$




$$
\begin{aligned}
= & \frac{1}{2 m(1-m) K(m)^{2}}\left(2 E(m)(1-m) K(m)-(1-m)^{2} K(m)^{2}-E(m)^{2}\right) \\
& +\frac{1}{2 m(1-m) K(m)^{2}}\left(\left((1-m)^{2}-(1-m)\right) K(m)^{2}\right) \\
= & \frac{1}{2 m(1-m) K(m)^{2}}\left(-(E(m)-(1-m) K(m))^{2}-m(1-m) K(m)^{2}\right) \\
\leq & -\frac{1}{2} .
\end{aligned}
$$

It remains to show that $m^{*}>\frac{1}{2}$. Indeed

$$
\begin{aligned}
2 E\left(\frac{1}{2}\right)-K\left(\frac{1}{2}\right) & =\int_{0}^{\frac{\pi}{2}}\left(2 \sqrt{1-\frac{1}{2} \sin ^{2}(\theta)}-\frac{1}{\sqrt{1-\frac{1}{2} \sin ^{2}(\theta)}}\right) \mathrm{d} \theta \\
& =\int_{0}^{\frac{\pi}{2}} \frac{\cos ^{2}(\theta)}{\sqrt{1-\frac{1}{2} \sin ^{2}(\theta)}} \mathrm{d} \theta>0,
\end{aligned}
$$

which implies that $f\left(\frac{1}{2}\right)>0$ and hence by monotonicity of $f$ we find $m^{*}>\frac{1}{2}$.

Lemma B.5. The expression $2 E(m)-K(m)+m K(m)$ is strictly positive for all $m \in$ $(0,1)$.

Proof. Let $f(m):=\frac{2 E(m)}{K(m)}-1+m$. Note note that $f(m)$ is positive if and only if the expression in the statement is positive and $K(m)>0$. Further note that

$$
\lim _{m \rightarrow 1} f(m)=0 .
$$

To show the claim it suffices to prove that $f^{\prime}<0$. To do so, it suffices to show that $\frac{\mathrm{d}}{\mathrm{d} m} \frac{E(m)}{K(m)}<-\frac{1}{2}$ for all $m \in(0,1)$. We have already shown in $(32)$ that

$\frac{\mathrm{d}}{\mathrm{d} m} \frac{E(m)}{K(m)}=\frac{1}{2 m(1-m) K(m)^{2}}\left(-(E(m)-(1-m) K(m))^{2}-m(1-m) K(m)^{2}\right) \leq-\frac{1}{2}$,

where the last inequality was obtained by estimating the square with zero. We will show that this estimate is always with strict inequality, i.e.

$$
E(m)-(1-m) K(m) \neq 0 \quad \forall m \in(0,1) .
$$

Note again first that

$$
\lim _{m \rightarrow 0}(E(m)-(1-m) K(m))=0 .
$$

Now an easy computation yields

$$
\frac{\mathrm{d}}{\mathrm{d} m}(E(m)-(1-m) K(m))=\frac{1}{2} K(m)>0 .
$$


Therefore we obtain that

$$
E(m)-(1-m) K(m)>0 \quad \forall m \in(0,1) .
$$

Hence (33) is shown and thus $\frac{d}{d m} \frac{E(m)}{K(m)}<-\frac{1}{2}$ for all $m \in(0,1)$. By definition of $f$ we obtain $f^{\prime}<0$.

Lemma B.6. Let $m^{*}$ be the unique zero in LemmaB.4. Then the map

$$
[0,2 \pi) \ni x \mapsto 2 E\left(x, m^{*}\right)-F\left(x, m^{*}\right)
$$

has exactly four zeroes in $[0,2 \pi)$, namely $x_{1}=0, x_{2}=\frac{\pi}{2}, x_{3}=\pi, x_{4}=\frac{3 \pi}{2}$.

Proof. Let $f: \mathbb{R} \rightarrow \mathbb{R}$ be the smooth function defined by $f(x):=2 E\left(x, m^{*}\right)-F\left(x, m^{*}\right)$. We show first that $f(0)=f\left(\frac{\pi}{2}\right)=f(\pi)=f\left(\frac{3 \pi}{2}\right)=f(2 \pi)$. Indeed, by Proposition B.3 one has

$$
f\left(l \frac{\pi}{2}\right)=l\left(2 E\left(m^{*}\right)-K\left(m^{*}\right)\right)=0 .
$$

Next we show that $f^{\prime}$ has four zeroes in $[0,2 \pi]$. Indeed,

$$
f^{\prime}(x)=\frac{1-2 m^{*} \sin ^{2}(x)}{\sqrt{1-m^{*} \sin ^{2}(x)}},
$$

which is zero if and only if $\sin ^{2}(x)=\frac{1}{2 m^{*}}$ which happens exactly four times in $[0,2 \pi]$ since $m^{*}>\frac{1}{2}$ by Lemma B.4. Assume now that there exists some $x_{0} \in(0,2 \pi)$ apart from $0, \frac{\pi}{2}, \pi, \frac{3 \pi}{2}, 2 \pi$ such that $f\left(x_{0}\right)=0$. We can now sort the set $\left\{0, \frac{\pi}{2}, \pi, \frac{3 \pi}{2}, 2 \pi, x_{0}\right\}=$ $\left\{y_{1}, y_{2}, y_{3}, y_{4}, y_{5}, y_{6}\right\}$ with $0=y_{1}<\ldots<y_{6}=2 \pi$. Since

$$
f\left(y_{1}\right)=\ldots=f\left(y_{6}\right)=0
$$

by the mean value theorem for all $i \in\{1, \ldots, 5\}$ there exists some $z_{i} \in\left(y_{i}, y_{i+1}\right)$ such that $f^{\prime}\left(z_{i}\right)=0$. This however is a contradiction to the fact that $f^{\prime}$ has only 4 zeroes. As a consequence, there exists no $x_{0}$ as in the assumption. The claim follows.

Lemma B.7 (cf. [MS20, Proposition B.5]). For all $m \in(0,1)$ one has

$$
E(m) \leq \frac{\pi}{2 \sqrt{2}} \sqrt{2-m}
$$

Proof. The proof follows from [MS20, Proposition B.5]. Be aware that the authors there use the different notation of $m=p^{2}$, their definition of $E(p)$ is actually $E\left(p^{2}\right)$ in our notation. 


\section{B.3 Explicit parametrization of Euler's elasticae}

In the following we shall prove the following classification result.

Proposition B.8. Let $I \subset \mathbb{R}$ be an interval and let $\gamma: I \rightarrow \mathbb{R}$ be a smooth solution of (7) for some $\lambda \in \mathbb{R}$. Then up to rescaling, reparametrization and isometries of $\mathbb{R}^{2}, \gamma$ is given by one of the following elastic prototypes.

1. (Linear elastica) $\gamma$ is a line, $\kappa[\gamma]=0$.

2. (Wavelike elastica) There exists $m \in(0,1)$ such that

$$
\gamma(s)=\left(\begin{array}{c}
2 E(\operatorname{am}(s, m), m)-s \\
-2 \sqrt{m} \mathrm{cn}(s, m)
\end{array}\right) .
$$

Moreover $\kappa[\gamma]=2 \sqrt{m} \mathrm{cn}(s, m)$.

3. (Borderline elastica)

$$
\gamma(s)=\left(\begin{array}{c}
2 \tanh (s)-s \\
-2 \operatorname{sech}(s)
\end{array}\right)
$$

Moreover $\kappa[\gamma]=2 \operatorname{sech}(s)$.

4. (Orbitlike elastica) There exists $m \in(0,1)$ such that

$$
\gamma(s)=\frac{1}{m}\left(\begin{array}{c}
2 E(\operatorname{am}(s, m), m)+(m-2) s \\
-2 \operatorname{dn}(s, m)
\end{array}\right)
$$

Moreover $\kappa[\gamma]=2 \operatorname{dn}(s, m)$.

5. (Circular elastica) $\gamma$ is a circle.

We give a proof in the rest of this section. Suppose that $\gamma$ is parametrized by arc-length. As we know that $\kappa$ satisfies (7), using [Lin96, Proposition 3.3] we obtain that one out of the four following cases occurs.

(1) (Constant curvature) $\kappa$ is constant.

(2) (Wavelike elastica) $\kappa(s)= \pm 2 \alpha \sqrt{m} \mathrm{cn}\left(\alpha\left(s-s_{0}\right), m\right.$ ) for some $m \in[0,1), \alpha>0, s_{0} \in$ $\mathbb{R}$. In this case $\lambda=\alpha^{2}(2 m-1)$.

(3) (Orbitlike elastica) $\kappa(s)= \pm 2 \alpha \operatorname{dn}\left(\alpha\left(s-s_{0}\right), m\right)$ for some $m \in[0,1), \alpha>0, s_{0} \in \mathbb{R}$. In this case $\lambda=\alpha^{2}(2-m)$.

(4) (Borderline elastica) $\kappa(s)= \pm 2 \alpha \operatorname{sech}\left(\alpha\left(s-s_{0}\right)\right.$ ) for some $\alpha>0, s_{0} \in \mathbb{R}$. In this case $\lambda=\alpha^{2}$. 
Once this is known we can find explicit parametrizations of all these elastica. Note that once we have parametrized the solutions for $s_{0}=0$ and ' + ' instead of ' \pm ' we can obtain all other solutions by reparametrization or reflection. Hence we consider only the cases of ' + ' and $s_{0}=0$.

From [DP17, Proposition 6.1] it is known that each smooth solution $\gamma: I \rightarrow \mathbb{R}^{2}$ of (7) with some parameter $\lambda \in \mathbb{R}$ corresponds up to isometries of $\mathbb{R}^{2}$ and reparametrization to a solution of

$$
\left\{\begin{array}{l}
\gamma_{1}^{\prime \prime}=\sigma \gamma_{2} \gamma_{2}^{\prime}, \\
\gamma_{2}^{\prime \prime}=-\sigma \gamma_{2} \gamma_{1}^{\prime} \\
\gamma_{1}^{\prime 2}+\gamma_{2}^{\prime 2}=1
\end{array}\right.
$$

for some $\sigma>0$. One can now compute that if $\gamma$ is a solution of (34) then $\kappa=\gamma_{2}^{\prime \prime} \gamma_{1}^{\prime}-$ $\gamma_{1}^{\prime \prime} \gamma_{2}^{\prime}=-\sigma \gamma_{2}$ and $\gamma_{1}^{\prime}-\frac{\sigma}{2} \gamma_{2}^{2} \equiv \mu$ for some constant $\mu \in \mathbb{R}$. Following the lines of [?, Proposition 6.1] we also obtain that $\lambda=-\sigma \mu$. We are also free to assume that $\gamma_{1}(0)=0$ as (34) is not affected by adding a constant to $\gamma_{1}$. From now on the parameters $\alpha$ and $m$ will be our main parameters. We will express $\sigma, \mu$ in terms of them and use (34) to obtain an explicit parametrization.

Case 1: Constant curvature. This yields either lines or circles.

Case 2: Wavelike elastica. First we show that $\sigma=\alpha^{2}$ and $\mu=1-2 m$. Note that by point (1) of the list of possible curvatures and $\lambda=-\sigma \mu$ we have that $\mu=\frac{\alpha^{2}}{\sigma}(1-2 m)$. In partcular, since $\kappa=-\sigma \gamma_{2}$ we have

$$
\gamma_{2}(s)=-\frac{2}{\sigma} \alpha \sqrt{m} \operatorname{cn}(\alpha s, m)
$$

and since $\gamma_{1}^{\prime}=\frac{\sigma}{2} \gamma_{2}^{2}+\mu$ we obtain

$$
\gamma_{1}^{\prime}(s)=\frac{\sigma}{2} \gamma_{2}(s)^{2}+\frac{\alpha^{2}}{\sigma}(1-2 m)=\frac{\alpha^{2}}{\sigma}\left(2 m \mathrm{cn}^{2}(\alpha s, m)+1-2 m\right) .
$$

Therefore using (34) and Proposition B.3 we obtain

$$
\begin{aligned}
1 & =\gamma_{1}^{\prime}(s)^{2}+\gamma_{2}^{\prime}(s)^{2} \\
& =\frac{\alpha^{4}}{\sigma^{2}}\left(\left(2 m \mathrm{cn}^{2}(\alpha s, m)+1-2 m\right)^{2}+4 m \operatorname{sn}^{2}(\alpha s, m) \operatorname{dn}^{2}(\alpha s, m)\right) \\
& =\frac{\alpha^{4}}{\sigma^{2}}\left(\left(1-2 m \operatorname{sn}^{2}(\alpha s, m)\right)^{2}+4 m \operatorname{sn}^{2}(\alpha s, m)\left(1-m \operatorname{sn}^{2}(\alpha s, m)\right)\right)=\frac{\alpha^{4}}{\sigma^{2}}
\end{aligned}
$$

Hence $\sigma=\alpha^{2}$, which implies by $(35)$ that

$$
\gamma_{2}(s)=-\frac{1}{\alpha} \sqrt{m} \operatorname{cn}(\alpha s, m) .
$$

We can moreover improve the formula for $\mu$ to $\mu=1-2 m$. Using this and (36) we find

$$
\gamma_{1}^{\prime}(s)=2 m \mathrm{cn}^{2}(\alpha s, m)+(1-2 m)=1-2 m \mathrm{sn}^{2}(\alpha s, m)
$$


and integrating using $\gamma_{1}(0)=0$ we obtain

$$
\begin{aligned}
\gamma_{1}(s) & =s-2 m \int_{0}^{s} \operatorname{sn}^{2}(\alpha s, m) \mathrm{d} s=s-\frac{2}{\alpha} \int_{0}^{\mathrm{am}(\alpha s, m)} \frac{m \sin ^{2} \theta}{\sqrt{1-m \sin ^{2} \theta}} d \theta \\
& =s-\frac{2}{\alpha} \int_{0}^{\mathrm{am}(\alpha s, m)}\left(\frac{1}{\sqrt{1-m \sin ^{2} \theta}}-\sqrt{1-m \sin ^{2} \theta}\right) d \theta \\
& =s-\frac{2}{\alpha} F(\operatorname{am}(\alpha s, m), m)+\frac{2}{\alpha} E(\operatorname{am}(\alpha s, m), m)=\frac{2}{\alpha}(E(\operatorname{am}(\alpha s, m), m)-\alpha s) .
\end{aligned}
$$

Hence for fixed $\alpha>0$ one has $\gamma(s)=\frac{1}{\alpha} \gamma_{\text {wave }}(\alpha s)$ where $\gamma_{\text {wave }}$ is given by

$$
\gamma_{\text {wave }}(s)=\left(\begin{array}{c}
2 E(\operatorname{am}(s, m), m)-s \\
-2 \sqrt{m} \mathrm{cn}(s, m)
\end{array}\right) .
$$

Case 3: Orbitlike elastica. We proceed as in the wavelike case. We first show that $\sigma=\alpha^{2} m$ and $\mu=\frac{m-2}{m}$. From point (2) in the list of curvatures and $\lambda=-\sigma \mu$ we infer that $\mu=\frac{\alpha^{2}(m-2)}{\sigma}$. This leads to

$$
\gamma_{2}(s)=-\frac{2}{\sigma} \alpha \operatorname{dn}(\alpha s, m)
$$

and by Proposition B.3

$$
\gamma_{1}^{\prime}(s)=\frac{\sigma}{2} \gamma_{2}(s)^{2}+\frac{\alpha^{2}(m-2)}{\sigma}=\frac{\alpha^{2} m}{\sigma}\left(1-2 \operatorname{sn}^{2}(\alpha s, m)\right)
$$

Using (34) and Proposition B.3 we obtain

$$
\begin{aligned}
1 & =\gamma_{1}^{\prime}(s)^{2}+\gamma_{2}^{\prime}(s)^{2} \\
& =\frac{\alpha^{4} m^{2}}{\sigma^{2}}\left(\left(1-2 \mathrm{sn}^{2}(\alpha s, m)\right)^{2}+4 \mathrm{sn}^{2}(\alpha s, m) \mathrm{cn}^{2}(\alpha s, m)\right) \\
& =\frac{\alpha^{4} m^{2}}{\sigma^{2}}\left(\left(1-2 \mathrm{sn}^{2}(\alpha s, m)\right)^{2}+4 \mathrm{sn}^{2}(\alpha s, m)\left(1-\operatorname{sn}^{2}(\alpha s, m)\right)=\frac{\alpha^{4} m^{2}}{\sigma^{2}}\right.
\end{aligned}
$$

Therefore we find that $\sigma=\alpha^{2} m$ and from this follows that $\mu=\frac{m-2}{m}$. We infer

$$
\gamma_{2}(s)=-\frac{2}{m \alpha} \operatorname{dn}(\alpha s, m)
$$

and

$$
\gamma_{1}^{\prime}(s)=1-2 \operatorname{sn}^{2}(\alpha s, m)
$$

Integrating we obtain

$$
\gamma_{1}(s)=s-2 \int_{0}^{s} \operatorname{sn}^{2}(\alpha s, m) \mathrm{d} s=s\left(1-\frac{2}{m}\right)+\frac{2}{\alpha m} E(\operatorname{am}(\alpha s, m), m) .
$$


We infer that for fixed $\alpha>0$ one has that $\gamma(s)=\frac{1}{\alpha} \gamma_{\text {orbit }}(\alpha s)$, where $\gamma_{\text {orbit }}$ is given by

$$
\gamma_{\text {orbit }}=\frac{1}{m}\left(\begin{array}{c}
2 E(\operatorname{am}(s, m), m)+(m-2) s \\
-2 \operatorname{dn}(s, m)
\end{array}\right) .
$$

Case 4: Borderline elastica One can proceed exactly as in the first two cases and obtain that for fixed $\alpha>0$ one has that $\gamma=\frac{1}{\alpha} \gamma_{\text {border }}(\alpha s)$ where

$$
\gamma_{b o r d e r}(s)=\left(\begin{array}{c}
2 \tanh (s)-s \\
-2 \operatorname{sech}(s)
\end{array}\right)
$$

\section{References}

[AS64] Milton Abramowitz and Irene A. Stegun. Handbook of mathematical functions with formulas, graphs, and mathematical tables, volume 55 of National Bureau of Standards Applied Mathematics Series. Dover Publications, New York, 1964.

[Bär10] Christian Bär. Elementary differential geometry. Cambridge University Press, Cambridge, 2010. Translated from the 2001 German original by P. Meerkamp.

[BH74] Frederick Brickell and Chuan-Chih Hsiung. The total absolute curvature of closed curves in Riemannian manifolds. J. Differential Geometry, 9:177-193, 1974 .

[DHMV08] Peter A. Djondjorov, Mariana Ts. Hadzhilazova, Ivaïlo M. Mladenov, and Vassil M. Vassilev. Explicit parameterization of Euler's elastica. In Geometry, integrability and quantization, pages 175-186. Softex, Sofia, 2008.

[DKS02] Gerhard Dziuk, Ernst Kuwert, and Reiner Schätzle. Evolution of elastic curves in $\mathbb{R}^{n}$ : existence and computation. SIAM J. Math. Anal., 33(5):1228$1245,2002$.

[DP17] Anna Dall'Acqua and Alessandra Pluda. Some minimization problems for planar networks of elastic curves. Geom. Flows, 2(1):105-124, 2017.

[DPS16] Anna Dall'Acqua, Paola Pozzi, and Adrian Spener. The Łojasiewicz-Simon gradient inequality for open elastic curves. J. Differential Equations, 2016.

[EG19] Sascha Eichmann and Hans-Christoph Grunau. Existence for Willmore surfaces of revolution satisfying non-symmetric Dirichlet boundary conditions. Adv. Calc. Var., 12(4):333-361, 2019.

[Fár49] István Fáry. Sur la courbure totale d'une courbe gauche faisant un nœud. Bull. Soc. Math. France, 77:128-138, 1949. 
[Fen29] Werner Fenchel. Über Krümmung und Windung geschlossener Raumkurven. Math. Ann., 101(1):238-252, 1929.

[Heb96] Emmanuel Hebey. Sobolev spaces on Riemannian manifolds, volume 1635 of Lecture Notes in Mathematics. Springer-Verlag, Berlin, 1996.

[Lee03] John M. Lee. Introduction to smooth manifolds, volume 218 of Graduate Texts in Mathematics. Springer-Verlag, New York, 2003.

[Lin96] Anders Linnér. Unified representations of nonlinear splines. J. Approx. Theory, 84(3):315-350, 1996.

[LS84a] Joel Langer and David A. Singer. Curves in the hyperbolic plane and mean curvature of tori in 3-space. Bull. London Math. Soc., 16(5):531-534, 1984.

[LS84b] Joel Langer and David A. Singer. Knotted elastic curves in $\mathbb{R}^{3}$. J. London Math. Soc. (2), 30(3):512-520, 1984.

[LS84c] Joel Langer and David A. Singer. The total squared curvature of closed curves. J. Differential Geom., 20(1):1-22, 1984.

[LY82] Peter Li and Shing Tung Yau. A new conformal invariant and its applications to the Willmore conjecture and the first eigenvalue of compact surfaces. Invent. Math., 69(2):269-291, 1982.

[Mil50] John W. Milnor. On the total curvature of knots. Ann. of Math. (2), 52:248$257,1950$.

[Mil53] John W. Milnor. On total curvatures of closed space curves. Math. Scand., 1:289-296, 1953.

[MP20] Carlo Mantegazza and Marco Pozzetta. The Lojasiewicz-Simon inequality for the elastic flow, 2020. arXiv:2007.16093.

[MS20] Marius Müller and Adrian Spener. On the Convergence of the Elastic Flow in the Hyperbolic Plane. Geom. Flows, 5(1):40-77, 2020.

[Poz20] Marco Pozzetta. A varifold perspective on the $p$-elastic energy of planar sets. J. Convex Anal., 27(3):845-879, 2020.

[RS20] Fabian Rupp and Adrian Spener. Existence and convergence of the lengthpreserving elastic flow of clamped curves, 2020. arXiv preprint.

[Rup20] Fabian Rupp. On the Łojasiewicz-Simon gradient inequality on submanifolds. J. Funct. Anal., 279(8):108708, 2020.

[Woj20] Stephan Wojtowytsch. Confined elasticae and the buckling of cylindrical shells. Advances in Calculus of Variations, 2020. Ahead of Print.

[Zei96] Eberhard Zeidler. Nonlinear Functional Analysis and its Applications: I: Fixed-Point Theorems. Springer, 3rd edition, 1996. 